Tohoku Math. J.

65 (2013), 131-157

\title{
A COMPARISON THEOREM FOR STEINER MINIMUM TREES IN SURFACES WITH CURVATURE BOUNDED BELOW
}

\author{
SHINTARO NAYA AND NOBUHIRO INNAMI
}

(Received October 19, 2010, revised May 7, 2012)

\begin{abstract}
Let $D$ be a compact polygonal Alexandrov surface with curvature bounded below by $\kappa$. We study the minimum network problem of interconnecting the vertices of the boundary polygon $\partial D$ in $D$. We construct a smooth polygonal surface $\widetilde{D}$ with constant curvature $\kappa$ such that the length of its minimum spanning trees is equal to that of $D$ and the length of its Steiner minimum trees is less than or equal to $D$ 's. As an application we show a comparison theorem of Steiner ratios for polygonal surfaces.
\end{abstract}

1. Introduction. Roughly speaking, a minimal geodesic segment is a curve whose length is equal to the distance between its endpoints. The distance plays very important roles in various researches. It means that the geometry of geodesics can be useful and have many applications. The minimum network problem is one of them. We will discuss the minimum network problem on surfaces from a geometric point of view. The theory of Alexandrov surfaces plays important roles because some comparison theorems are very useful to make a suitable configuration of points in a model surface with constant curvature.

Let $M$ be a complete Alexandrov surface with curvature bounded below by $\kappa$ having no boundary. Examples of those surfaces are complete 2-dimensional Riemannian manifolds with curvature bounded below by $\kappa$ having no boundary, convex surfaces without boundary in the Euclidean space $\boldsymbol{E}^{3}$, glued surfaces with curvature bounded below by $\kappa$ having no boundary [8], [16], [17]. Let $P$ be a finite set of points in $M$. A shortest network interconnecting $P$ is called a Steiner minimum tree which is denoted by $\operatorname{SMT}_{M}(P)$. A Steiner minimum tree $\mathrm{SMT}_{M}(P)$ may have vertices which are not in $P$. Such vertices are called Steiner points. A spanning tree on $P$ is by definition a tree interconnecting $P$ with vertex set $P$, so that all edges are minimal geodesic segments connecting two points in $P$. A shortest spanning tree on $P$ is called a minimum spanning tree on $P$ which is denoted by $\operatorname{MST}_{M}(P)$. If $P$ consists of two points $p$ and $q$ in $M$, then $\operatorname{SMT}_{M}(P)$ and $\operatorname{MST}_{M}(P)$ are a minimal geodesic segment $T(p, q)$. Let $L(T)$ be the total length of edges in a tree $T$. The Steiner ratio $\rho$ for $M$ is given by

$$
\rho=\rho(M)=\inf _{P} \frac{L\left(\operatorname{SMT}_{M}(P)\right)}{L\left(\operatorname{MST}_{M}(P)\right)}
$$

2000 Mathematics Subject Classification. Primary 53C20; Secondary 05 C05.

Key words and phrases. Steiner tree, geodesic, Alexandrov space.

Research of the second author was partially supported by Grant-in-Aid for Scientific Research (C), 22540072. 
where $P$ runs over all finite sets of more than two points in $M$. If $M$ is the Euclidean plane $\boldsymbol{E}^{2}$ and $P$ consists of three points which make an equilateral triangle, then we have the ratio

$$
\frac{L\left(\operatorname{SMT}_{\boldsymbol{E}^{2}}(P)\right)}{L\left(\mathrm{MST}_{\boldsymbol{E}^{2}}(P)\right)}=\frac{\sqrt{3}}{2} .
$$

Du and Hwang [6] stated that $\rho(M)=\sqrt{3} / 2$ if $M$ is the Euclidean plane. This was the affirmative answer of a famous conjecture of Gilbert and Pollak [7]. However, Ivanov and Tuzhilin [12], de Wet [19] and Innami et al. [11] have recently pointed out that their proof is not complete. Rubinstein and Weng [15] have stated that $\rho(M)=\sqrt{3} / 2$ if $M$ is a 2 dimensional sphere of constant curvature. However, their proof used the result stated in [6], so the proof is not complete. Ivanov, Tuzhilin and Cieslik [13] have estimated some Steiner ratios for manifolds. Innami and $\operatorname{Kim}[10$ ] have proved that $\rho(M)=1 / 2$ if $M$ is a complete simply connected surface of negative constant curvature and without boundary. Tamura and Innami [18] succeeded in generalizing their theorem, proving that $\rho(M) \leq n / 2(n-1)$ if $M$ is a complete locally compact length space with $n$ ends.

Rubinstein and Weng [15] expected that the Toponogov comparison theorem is useful to generalize their theorems. The present paper shows that their expectation is right.

We say that a simply connected compact surface $D$ with vertex set $v(\partial D)$ is a polygonal surface with curvature bounded below by $\kappa$ if Int $D$ is a locally compact Alexandrov surface with curvature bounded below by $\kappa$ and the boundary $\partial D$ is the union of minimal geodesic segments $T\left(p_{i}, p_{i+1}\right)$ in $D$ connecting adjacent vertices $p_{i}, p_{i+1} \in v(\partial D)$ which are locally convex. We will see the definition of an Alexandrov surface with curvature bounded below by $\kappa$ in Section 2. Let Int $T=T \backslash\{p, q\}$ for a minimal geodesic segment $T=T(p, q)$ connecting points $p, q \in D$. Here, a minimal geodesic segment $T$ contained in $\partial D$ is by definition locally convex if for any point $p \in \operatorname{Int} T$ there exists a positive $\varepsilon$ such that any minimal geodesic segment $T_{1}$ with $p \in \operatorname{Int} T_{1}$ in the $\varepsilon$-ball around $p$ in $D$ is a subsegment of $T$, namely $T_{1} \subset T$. The total angle around at any point in Int $D$ is less than or equal to $2 \pi$. A point in Int $D$ having total angle less than $2 \pi$ is said to be singular. If there is no singular point in Int $D$, then $D$ is said to be smooth. The inner angle around any point in $\partial D \backslash v(\partial D)$ is $\pi$. However, the inner angle at a vertex $p \in v(\partial D)$ may be greater than $2 \pi$. We call such a vertex in $v(\partial D)$ a spiral vertex. Let $\operatorname{SMT}(D)$ and $\operatorname{MST}(D)$ denote a Steiner minimum tree for $v(\partial D)$ in $D$ and a minimum spanning tree on $v(\partial D)$ in $D$, respectively. In general, $\operatorname{SMT}(D)$ and $\operatorname{MST}(D)$ are not uniquely determined.

The first purpose of this paper is to prove the following theorem.

THEOREM 1. Let D be a polygonal surface with curvature bounded below by $\kappa$. Then, there exists a smooth polygonal surface $\widetilde{D}$ with constant curvature $\kappa$ such that

$$
L(\operatorname{SMT}(D)) \geq L(\operatorname{SMT}(\widetilde{D})) \text { and } L(\operatorname{MST}(D))=L(\operatorname{MST}(\widetilde{D})) .
$$

In particular, we have

$$
\frac{L(\operatorname{SMT}(D))}{L(\operatorname{MST}(D))} \geq \frac{L(\operatorname{SMT}(\widetilde{D}))}{L(\operatorname{MST}(\widetilde{D}))}
$$


We do not assume that a polygonal surface $D$ can be immersed into any Alexandrov surface. However, if $\widetilde{D}$ is smooth and flat, then $\widetilde{D}$ is immersed into the Euclidean plane $\boldsymbol{E}^{2}$ (see Lemma 25).

We define a new ratio to estimate the Steiner ratio. Let $M$ be a simply connected complete Alexandrov surface with curvature bounded below by $\kappa$ having no boundary. Let $D$ be an immersed polygonal surface in $M$. Let $\operatorname{SMT}(D)$ be a Steiner minimum tree for $v(\partial D)$ in $D$ and $\operatorname{MST}(D)$ a minimum spanning tree on $v(\partial D)$ in $D$. We define the Steiner ratio $\eta$ of $M$ for immersed polygonal surfaces by

$$
\eta=\eta(M)=\inf _{D} \frac{L(\operatorname{SMT}(D))}{L(\operatorname{MST}(D))},
$$

where $D$ runs over all immersed polygonal surfaces in $M$. Du and Hwang [6] have given an idea to construct immersed polygonal surfaces which are called the characteristic areas $C(T)$ for any full Steiner trees $T$ in the Euclidean plane $\boldsymbol{E}^{2}$. Ivanov and Tuzhilin [12] actually succeeded in constructing them in a different way. Since they are immersed polygonal surfaces, we have

$$
\rho\left(\boldsymbol{E}^{2}\right) \geq \inf _{T} \frac{L(\operatorname{SMT}(C(T)))}{L(\operatorname{MST}(C(T)))} \geq \eta\left(\boldsymbol{E}^{2}\right)
$$

where $T$ runs over all full Steiner trees in $\boldsymbol{E}^{2}$.

Their construction of characteristic areas are valid in a domain $M_{0} \subset M$ if there is the unique minimal geodesic segments connecting their endpoints in $M_{0}$. We say that a domain $M_{0}$ is convex if for any $p, q \in M_{0}$ there exists a minimal geodesic segment connecting $p$ and $q$ in $M$ which is contained in $M_{0}$. We say that a convex domain is strong if the minimal geodesic segment $T(p, q)$ connecting $p$ and $q$ in $M_{0}$ is unique in $M_{0}$. Any strongly convex domain in an Alexandrov surface with curvature bounded below by $\kappa$ contains no singular point. The open hemispheres are examples of strongly convex domains. In a smooth Riemannian surface any sufficiently small ball around any point is strongly convex.

As an application of Theorem 1 we prove the following.

THEOREM 2. Let $M$ be a complete Alexandrov surface with nonnegative curvature having no boundary and $M_{0}$ a strongly convex domain in $M$. Then, we have

$$
\frac{\sqrt{3}}{2} \geq \rho\left(M_{0}\right) \geq \eta\left(M_{0}\right) \geq \eta\left(\boldsymbol{E}^{2}\right)
$$

This inequality suggests a comparison theorem for Steiner ratios.

Here is the idea of our proof of Theorem 1. Alexandrov [1] introduced the cutting and pasting method to change a polygonal surface $D$ in the class of those surfaces with curvature bounded below by $\kappa$. Using his method, we make a deformation under which the length of MST does not change and the length of SMT does not increase. We deform the polygonal surface $D$ until it has constant curvature. Rubinstein and Weng [15] succeeded in doing this for spheres by using spheres with little by little increasing radii until it becomes flat. We cannot adopt spheres for modification in our case. Instead, we replace a small part of the polygonal surface $D$ by a piece of the corresponding domain with constant curvature $\kappa$ little 
by little until it is smooth and has constant curvature $\kappa$. This modification is possible in the class of Alexandrov surfaces with curvature bounded below by $\kappa$. To complete the gradual modification, we consider the partial ordered set $\mathcal{M}$ of polygonal surfaces $D_{\alpha}, \alpha \in \Lambda$, with curvature bounded below by $\kappa$, such that $D_{\alpha}$ are made of the original polygonal surface $D$ by compression maps given in Lemmas 5 and 6 . The main part of the proof is to construct an upper bound of any totally ordered subset $\mathcal{M}_{0}$ of $\mathcal{M}$. Then, we apply Zorn's lemma to have a maximum element $\widetilde{D}$ of $\mathcal{M}$. The maximum element $\widetilde{D}$ of $\mathcal{M}$ is a smooth polygonal surface satisfying the condition in Theorem 1.

The idea of the proof of Theorem 2 is the following. We first construct the characteristic area for a Steiner minimum tree in $M_{0}$ in the light of the idea of Du and Hwang [6]. The characteristic area is an immersed polygonal surface $D$ in $M_{0}$. We then have the second inequality. Thus, we apply Theorem 1 to estimate the ratio $\eta\left(M_{0}\right)$. We deform $D$ until it is smooth and flat. In the course of the modification, the polygonal surface $D$ is probably not immersed in any Alexandrov surface because points overlapping each other may have their neighborhoods which are not isometric. However, a smooth flat polygonal surface is immersed in the Euclidean plane $\boldsymbol{E}^{2}$. This shows the third inequality.

2. Alexandrov surfaces. Let $M$ be a locally compact space with intrinsic metric $d$. Then, every point $p$ in $M$ has a neighborhood $U(p)$ such that there exists a minimal curve connecting any points $q$ and $r$ in $U(p)$ whose length is equal to the distance between them. In addition, if $M$ is complete, then there exists a minimal curve $T(q, r)$ connecting any points $q$ and $r$ in $M$. Such a minimal curve is called a minimal geodesic segment $T(q, r)$ connecting $q$ and $r$. Moreover, there exists a shortest network interconnecting any finite set $P$ of points in $M$ which is called a Steiner minimum tree for $P$.

Let $M(\kappa)$ denote a simply connected complete surface with constant Gaussian curvature $\kappa$. If $\kappa$ is positive, zero and negative, then $M(\kappa)$ is isometric to the sphere with radius $1 / \sqrt{\kappa}$, the Euclidean plane $\boldsymbol{E}^{2}$ and the hyperbolic plane with curvature $\kappa$, respectively.

Let $\triangle_{1}(p q r)=T(p, q) \cup T(q, r) \cup T(p, r)$ be a geodesic triangle in $M$. We say that a geodesic triangle $\widetilde{\triangle}_{1}(p q r)=T(\tilde{p}, \tilde{q}) \cup T(\tilde{q}, \tilde{r}) \cup T(\tilde{p}, \tilde{r})$ in $M(\kappa)$ is a comparison triangle corresponding to $\triangle_{1}(p q r)$ if the lengths of its sides are the same as those of $\triangle_{1}(p q r)$. Here, the points $\tilde{p}, \tilde{q}, \tilde{r}$ denote the corresponding vertices of $\widetilde{\triangle}_{1}(p q r)$ to $p, q$, and $r$, respectively. Namely, $\widetilde{\triangle}_{1}(p q r)=\triangle_{1}(\tilde{p} \tilde{q} \tilde{r})$. The symbol $\tilde{p}, \tilde{q}, \tilde{r}$ are only used to denote the vertices of a comparison triangle corresponding to $\Delta_{1}(p q r)$. We say that a locally compact space $M$ with intrinsic metric $d$ is an Alexandrov space with curvature bounded below by $\kappa$ if $M$ satisfies the following condition. There exists a neighborhood $U(w)$ around any point $w$ in $M$ such that if $\triangle_{1}(p q r)$ is an arbitrary geodesic triangle in $U(w)$ and $\widetilde{\triangle}_{1}(p q r)$ is a comparison triangle corresponding to $\triangle_{1}(p q r)$ in $M(\kappa)$, and if $\kappa$ is positive we assume that the perimeter of $\triangle_{1}(p q r)$ is less than $2 \pi / \sqrt{\kappa}$, then the following is true.

(A) If $x \in T(q, r), x^{\prime} \in T(\tilde{q}, \tilde{r})$ with $d(q, x)=d\left(\tilde{q}, x^{\prime}\right)$, then $d(p, x) \geq d\left(\tilde{p}, x^{\prime}\right)$.

Let $x \in T(p, q)$ and $y \in T(p, r)$. Let $\omega(x, y)$ be the angle at $\tilde{p}$ of a comparison triangle $\triangle_{1}(\tilde{x} \tilde{p} \tilde{y})$. The angle $L(q p r)$ of $T(p, q)$ with $T(p, r)$ at $p$ is defined by $\angle(q p r)=$ 
$\lim _{x \rightarrow p, y \rightarrow p} \omega(x, y)$ if the limit exists. In an Alexandrov space with curvature bounded below by $\kappa$, this limit exists as seen in Lemma 3 (B). It follows from the definition that $\angle\left(q_{1} p r_{1}\right)$ is equal to $\angle(q p r)$ for any $q_{1} \in \operatorname{Int} T(p, q)$ and $r_{1} \in \operatorname{Int} T(p, r)$. We can see some conditions equivalent to $(\mathrm{A})$ in [2], [5].

LEMMA 3. The condition (A) is equivalent to the following conditions.

$\left(\mathrm{A}^{\prime}\right)$ If $x \in T(p, q), y \in T(p, r), x^{\prime} \in T(\tilde{p}, \tilde{q})$ and $y^{\prime} \in T(\tilde{p}, \tilde{r})$ with $d(p, x)=$ $d\left(\tilde{p}, x^{\prime}\right)$ and $d(p, y)=d\left(\tilde{p}, y^{\prime}\right)$, then $d(x, y) \geq d\left(x^{\prime}, y^{\prime}\right)$.

(B) The angle $\omega(x, y)$ at $\tilde{p}$ of a comparison triangle $\triangle_{1}(\tilde{p} \tilde{x} \tilde{y})$ corresponding to $\triangle_{1}(p x y)$ is monotone nonincreasing for $d(p, x)$ and $d(p, y)$ when $x \in T(p, q)$ and $y \in T(p, r)$.

(C) $L(p q r) \geq L(\tilde{p} \tilde{q} \tilde{r}), L(q r p) \geq L(\tilde{q} \tilde{r} \tilde{p})$ and $L(r p q) \geq L(\tilde{r} \tilde{p} \tilde{q})$.

(D) The sum of the angles at $\tilde{p}$ of comparison triangles $\triangle_{1}(\tilde{p} \tilde{q} \tilde{r}), \triangle_{1}(\tilde{p} \tilde{r} \tilde{s}), \triangle_{1}(\tilde{p} \tilde{s} \tilde{q})$ for any points $p, q, r, s$ is less than or equal to $2 \pi$.

(H) If $d(p, q)=d\left(p^{\prime}, q^{\prime}\right), d(p, r)=d\left(p^{\prime}, r^{\prime}\right)$ and $\angle(q p r)=L\left(q^{\prime} p^{\prime} r^{\prime}\right)$, then $d(q, r) \leq d\left(q^{\prime}, r^{\prime}\right)$. Here $p, q, r \in M$ and $p^{\prime}, q^{\prime}, r^{\prime} \in M(\kappa)$.

In $(\mathrm{H})$ we call the triple $(d(p, q), d(p, r), L(q p r))$ a hinge in $M$. If $M$ is complete, those properties mentioned in the definition and Lemma 3 are true for any geodesic triangle in $M$ and its comparison triangle in $M(\kappa)$.

The Hausdorff dimension $m$ of an Alexandrov space $M$ is equal to its topological dimension. If $m \leq 2$, then $M$ is a topological manifold with dimension $m$. We say that a point $p$ in $M$ is a singular point if the total angle around $p$ is less than $2 \pi$. Any minimal geodesic segment does not pass through a singular point in $M$ which is not its endpoint. Two minimal geodesic segments with the same endpoints do not intersect at any other point. Moreover, if $T(p, q)$ and $T(p, r)$ are minimal geodesic segments for $q \neq r \in M$, then $T(p, q) \cap T(p, r)=\{p\}$ unless one of them contains the other.

Let $M$ be a simply connected Alexandrov surface with curvature bounded below by $\kappa$. We say that $\triangle(p q r)$ is a geodesic triangle domain for points $p, q, r \in M$ if $\triangle(p q r)$ is a simply connected domain surrounded by $\triangle_{1}=T(p, q) \cup T(q, r) \cup T(r, p)$. The geodesic triangle domain may not be uniquely determined by its vertices. We say that a geodesic triangle domain $\widetilde{\triangle}(p q r)$ in $M(\kappa)$ is a comparison triangle domain corresponding to $\triangle(p q r)$ if the lengths of its sides are the same as those of $\Delta(p q r)$. When $\kappa>0$, there are two comparison triangle domains in $M(\kappa)$ with same vertices. Then, we accept the geodesic triangle contained in some hemisphere as $\widetilde{\triangle}(p q r)$.

We see the following lemma in [9].

LEMMA 4. Let $D$ be a polygonal surface with curvature bounded below by $\kappa$. Let $\triangle(p q r)$ be a geodesic triangle domain in $D$ whose inner angles are less than $\pi$ and $\widetilde{\triangle}(p q r)$ a comparison triangle domain corresponding to $\triangle(p q r)$ in $M(\kappa)$. If $\kappa$ is positive we assume that the perimeter of $\triangle(p q r)$ is less than $2 \pi / \sqrt{\kappa}$. Then, there exists a surjective map $\varphi$ : $\triangle(p q r) \rightarrow \widetilde{\triangle}(p q r)$ such that the restriction to each side is an isometry to the corresponding side, and $d(\varphi(x), \varphi(y)) \leq d(x, y)$ for any points $x, y \in \Delta(p q r)$. 
We say that a surjective map $\varphi: D \rightarrow \widetilde{D}$ is a compression map from $D$ onto $\widetilde{D}$ if the restriction of $\varphi$ to $\partial D$ preserves the arc length of $\partial D$ and $d(\varphi(x), \varphi(y)) \leq d(x, y)$ for any $x, y \in D$. The map $\varphi$ in Lemma 4 is a compression map. The idea of the proof of Lemma 4 shows us the fundamental technique for the deformation which will be used in the proof of Theorem 1.

THE IDEA OF THE PROOF. The map $\varphi$ is a combination of the following shrinking maps.

(1) Let $B$ be a subset of $D$ and $p^{\prime}$ a point in $B$. Let $\varphi_{1}(x)=p^{\prime}$ for all $x \in B$. Then, $d\left(\varphi_{1}(x), \varphi_{1}(y)\right) \leq d(x, y)$ for any $x, y \in B$ because the left hand side is always zero.

(2) Let $V(p, q)$ be a geodesic biangle domain with vertices $p$ and $q$ in $D$, namely, $V(p, q)$ is a domain in $D$ surrounded by two minimal geodesic segments $T_{1}=$ $T_{1}(p, q)$ and $T_{2}(p, q)$ connecting $p$ and $q$. Let $x_{1}=\varphi_{2}(x) \in T_{1}$ be the point with $d\left(p, x_{1}\right)=d(p, x)$ if $d(p, x) \leq d(p, q)$, otherwise $x_{1}=q$ for any $x \in V(p, q)$. Then, $d\left(\varphi_{2}(x), \varphi_{2}(y)\right) \leq d(x, y)$ for any $x, y \in V(p, q)$ because of the triangle inequality of the distance $d$.

(3) Let $C(p, a)=\{x \in D ; d(p, x)=a\}$ and $S(p ; q, r)$ the sector surrounded by $T_{1}=T(p, q), T(p, r)$ and the subarc of $C(p, a)$ connecting $q$ and $r$. Let $x_{1}=$ $\varphi_{3}(x) \in T_{1}$ be the point with $d\left(p, x_{1}\right)=d(p, x)$ if $d(p, x) \leq a$, otherwise $x_{1}=q$ for any $x \in S(p ; q, r)$. Then, $d\left(\varphi_{3}(x), \varphi_{3}(y)\right) \leq d(x, y)$ for any $x, y \in S(p ; q, r)$ because of the triangle inequality of the distance $d$.

(4) Let $\triangle_{1}(p q r)$ be a geodesic triangle whose inner angles are less than $\pi$ and $\widetilde{\triangle}_{1}(p q r)$ the comparison triangle corresponding to $\triangle_{1}(p q r)$. Let $\varphi_{4}(x)$ be the corresponding point $x^{\prime}$ in $\widetilde{\triangle}_{1}(p q r)$ for any $x \in \triangle_{1}(p q r)$. Then, $d\left(\varphi_{4}(x), \varphi_{4}(y)\right) \leq$ $d(x, y)$ for any $x, y \in \Delta_{1}(p q r)$ because of the condition $\left(\mathrm{A}^{\prime}\right)$ in Lemma 3.

Using these shrinking maps, we construct the compression map $\varphi$ as follows. Let $m$ and $m^{\prime}$ be the midpoints of $T(q, r)$ and $T(\tilde{q}, \tilde{r})$, respectively. By the condition (A) we have $d(p, m) \geq d\left(\tilde{p}, m^{\prime}\right)$. Then we find points $r_{1}$ and $q_{1}$ in $\triangle(p q r)$ such that $d\left(p, r_{1}\right)=d\left(\tilde{p}, m^{\prime}\right)$, $d\left(q, r_{1}\right)=d\left(\tilde{q}, m^{\prime}\right), d\left(p, q_{1}\right)=d\left(\tilde{p}, m^{\prime}\right)$, and $d\left(r, q_{1}\right)=d\left(\tilde{r}, m^{\prime}\right)$. Set $\Delta_{1}=\Delta(p q r) \backslash$ (Int $\triangle\left(p q r_{1}\right) \cup$ Int $\left.\triangle\left(p r q_{1}\right)\right)$. Then, $\Delta_{1}$ is divided into four pieces $S\left(p ; r_{1}, q_{1}\right), S\left(q ; m, r_{1}\right)$, $S\left(r ; m, q_{1}\right)$ and the remaining domain $B$. By using the above maps, we have a shrinking map from $\Delta_{1}$ onto $T(\tilde{p}, \tilde{q}) \cup T(\tilde{p}, \tilde{r}) \cup T(\tilde{q}, \tilde{r}) \cup T\left(\tilde{p}, m^{\prime}\right)$ in $\widetilde{\triangle}(p q r)$. Repeat this construction of shrinking maps for the remaining triangles $\triangle\left(p q r_{1}\right)$ and $\triangle\left(p r q_{1}\right)$ until we at last have a set $D_{2} \subset \triangle(p q r)$ such that $\triangle(p q r) \backslash D_{2}$ consists of at most countably many open geodesic biangle domains with vertex $p$, because those remaining triangle domains converge to minimal geodesic segments or geodesic biangle domains. We also have a shrinking map from $D_{2}$ into $\widetilde{\triangle}(p q r)$. Using the map in (2), we can make a shrinking map from each geodesic biangle domain of $\triangle(p q r) \backslash D_{2}$ onto a minimal geodesic segment. Combining these maps, we have a compression map from $\triangle(p q r)$ onto $\widetilde{\Delta}(p q r)$. 
We need to use the following lemma to change polygonal surfaces in the proof of Theorem 1. Alexandrov [1] shows the first parts of Lemmas 5 and 6 . However, we prove that Lemmas $3(\mathrm{C})$ is satisfied because we refer to the similar construction in the proof of Lemma 10.

Lemma 5. Let D be a polygonal surface with curvature bounded below by $\kappa$ and let $\triangle(p q r)$ be a geodesic triangle domain in Int $D$ whose inner angles are less than $\pi$. Let $\widetilde{\triangle}(p q r)$ be a comparison triangle domain corresponding to $\triangle(p q r)$ in $M(\kappa)$. Set $\bar{D}=(D \backslash$ $\triangle(p q r)) \cup \widetilde{\triangle}(p q r)$ where $\triangle(p q r)$ is removed from $D$ and then $\widetilde{\triangle}(p q r)$ is glued on $D \backslash \triangle(p q r)$ along corresponding sides. Assume that the distances between the adjacent vertices in $\partial D$ do not change. Then, $\bar{D}$ is also a polygonal surface with curvature bounded below by $\kappa$. There exists a compression map $\varphi: D \rightarrow \bar{D}$ such that the restriction to each side is an isometry to the corresponding side and $d(\varphi(x), \varphi(y)) \leq d(x, y)$ for any points $x, y \in D$.

It should be noted from the proof below that $d(\varphi(x), \varphi(y))=d(x, y)$ for $x$ and $y$ in $D$ if a minimal geodesic segment $T(\varphi(x), \varphi(y))$ does not intersect $\widetilde{\triangle}(p q r)$ in $\bar{D}$ because the distance is intrinsic. Equivalently, if $d(\varphi(x), \varphi(y))<d(x, y)$, then all minimal geodesic segments connecting $\varphi(x)$ and $\varphi(y)$ intersect $\widetilde{\triangle}(p q r)$ in $\bar{D}$. Thus, if all minimal geodesic segments $T=T(x, y)$ connecting $x$ and $y$ in $D$ do not pass through $p \in D$, we can make a polygonal surface $\bar{D}$ from $D$ such that $\varphi(T)=T(\varphi(x), \varphi(y))=T(x, y)$ which does not pass through $\varphi(p)$ in $\bar{D}$. In particular, if the minimal geodesic segments $T\left(p_{i}, p_{i+1}\right)$ connecting adjacent vertices $p_{i}, p_{i+1}$ in the vertex set $v(\partial D)$ are unique, then for any point $q \in \operatorname{Int} D$ there exists a sufficiently small convex geodesic triangle domain $\triangle(p q r)$ in Int $D$ satisfying the assumption in Lemma 5. This ensures that the edges in $\partial D$ are minimal geodesic segments in $\bar{D}$.

Proof. It is clear that $\bar{D}$ is simply connected and compact, and that $\partial \bar{D}$ is locally convex because of the assumption. It suffices to prove that Int $\bar{D}$ is an Alexandrov surface with curvature bounded below by $\kappa$. Let $w$ be a point in $\bar{D}$. If $w$ is not in the boundary $\partial \triangle(p q r)$ of $\triangle(p q r)$, then we can have a neighborhood around $w$ satisfying the condition (C) because $D \backslash \triangle(p q r)$ and Int $\triangle(\tilde{p} \tilde{q} \tilde{r})$ are Alexandrov surfaces with curvature bounded below by $\kappa$. Suppose $w \in \operatorname{Int} T(p, q) \cup \operatorname{Int} T(q, r) \cup \operatorname{Int} T(r, p)$, say $w \in \operatorname{Int} T(p, q)$. Let $U(w)$ be a neighborhood of $w$ in $\bar{D}$ which is divided by $\operatorname{Int} T(p, q)$ into two parts such that each component is contained in either $\triangle(\tilde{p} \tilde{q} \tilde{r})$ or $D \backslash \operatorname{Int} \triangle(p q r)$. This is possible because $T(p, q)$ is a minimal geodesic segment in both $\triangle(\tilde{p} \tilde{q} \tilde{r})$ and $D \backslash \operatorname{Int} \triangle(p q r)$. Let $\triangle(a b c)$ be a geodesic triangle domain in $U(w)$. All the cases we treat are proved in a similar way, so we suppose that $a \in \operatorname{Int} \triangle(\tilde{p} \tilde{q} \tilde{r})$ and $b, c \in \operatorname{Int}(D \backslash \operatorname{Int} \triangle(p q r))$, meaning that $T(a, b) \cap T(p, q)=\{u\}$ and $T(a, c) \cap T(p, q)=\{v\}$ for some points $u, v$.

We first prove that $L(a v u)+L(c v u)=\pi$. Suppose this is not true, namely, $L(a v u)+$ $L(c v u)<\pi$. Let $x \in T(a, v)$ and $y \in T(c, v)$ be sufficiently near $v$. Let $x^{\prime}, y^{\prime}$ and $v^{\prime}$ be points in $M(\kappa)$ such that $L\left(x^{\prime} v^{\prime} y^{\prime}\right)=L(a v u)+L(c v u), d\left(x^{\prime}, v^{\prime}\right)=d(x, v)$ and $d\left(y^{\prime}, v^{\prime}\right)=d(y, v)$. Take a point $z^{\prime} \in T\left(x^{\prime}, y^{\prime}\right)$ such that $T\left(z^{\prime}, v^{\prime}\right)$ makes angles $\angle(a v u)$ with $T\left(x^{\prime}, v^{\prime}\right)$ and $L(c v u)$ with $T\left(y^{\prime}, v^{\prime}\right)$, respectively. Let $z$ be the point in $T(p, q)$ with 


$$
\begin{aligned}
& d(v, z)=d\left(v^{\prime}, z^{\prime}\right)>0 \text {. Then, } \\
& \qquad \begin{aligned}
d(x, y) & =d(x, v)+d(v, y) \\
& =d\left(x^{\prime}, v^{\prime}\right)+d\left(v^{\prime}, y^{\prime}\right)>d\left(x^{\prime}, y^{\prime}\right) \\
& =d\left(x^{\prime}, z^{\prime}\right)+d\left(z^{\prime}, y^{\prime}\right) \geq d(x, z)+d(z, y)>d(x, y) .
\end{aligned}
\end{aligned}
$$

We use the comparison theorem of hinges in Lemma $3(\mathrm{H})$ to prove the second inequality. This is a contradiction.

Next we have $L(u a c) \geq L(\tilde{u} \tilde{a} \tilde{c})$ because the angle at $\tilde{v}$ of a quadrangle $\tilde{a} \tilde{u} \tilde{c} \tilde{v}$ is less than or equal to $\pi$ where the quadrangle $\tilde{a} \tilde{u} \tilde{c} \tilde{v}$ consists of two comparison triangle domains $\triangle(\tilde{a} \tilde{u} \tilde{v})$ and $\triangle(\tilde{c} \tilde{u} \tilde{v})$. By the same reasoning, we have $L(u c a) \geq L(\tilde{u} \tilde{c} \tilde{a})$.

Next we prove that $\angle(a u c) \geq \angle(\tilde{a} \tilde{u} \tilde{c})$. We claim that there exists a point $x \in T(u, v)$ such that the angle at $\tilde{x}$ of a quadrangle $\tilde{a}^{\prime} \tilde{u}^{\prime} \tilde{c}^{\prime} \tilde{x}$ is just equal to $\pi$ where the quadrangle $\tilde{a}^{\prime} \tilde{u}^{\prime} \tilde{c}^{\prime} \tilde{x}$ consists of two comparison triangle domains $\triangle\left(\tilde{a}^{\prime} \tilde{u}^{\prime} \tilde{x}\right)$ and $\triangle\left(\tilde{c}^{\prime} \tilde{u}^{\prime} \tilde{x}\right)$. This is proved as follows. As $x \in T(u, v)$ moves from $u$ to $v$, the angle at $\tilde{x}$ changes continuously because so do the distances from $x$ to $u$ and $v$. The inner angle of the quadrangle $\tilde{a}^{\prime} \tilde{u}^{\prime} \tilde{c}^{\prime} \tilde{x}$ at $\tilde{x}$ is greater than $\pi$ when $x$ is near $u$ and is less than $\pi$ when $x=v$. The mean value theorem ensures our claim. The quadrangle $\tilde{a}^{\prime} \tilde{u}^{\prime} \tilde{c}^{\prime} \tilde{x}$ is just a triangle domain $\triangle$ with its base containing $\tilde{x}$ which may not be the comparison triangle domain corresponding to $\triangle(a u c)$. Let $\triangle(\tilde{a} \tilde{u} \tilde{c})$ denote the comparison triangle domain corresponding to $\triangle(a u c)$. Since the base of $\triangle$ is longer than or equal to $T(a, c)$, we have

$$
\begin{aligned}
\angle(a u c) & =\angle(a u v)+\angle(c u v) \\
& =\angle(a u x)+\angle(c u x) \geq \angle\left(\tilde{a}^{\prime} \tilde{u}^{\prime} \tilde{x}\right)+\angle\left(\tilde{c}^{\prime} \tilde{u}^{\prime} \tilde{x}\right) \\
& \geq \angle(\tilde{a} \tilde{u} \tilde{c}) .
\end{aligned}
$$

We have used the Toponogov comparison theorem in Lemma 3 (C) for proving the first inequality $\geq$ and the hinge comparison theorem in Lemma $3(\mathrm{H})$ for the second one.

Next we have $\angle(b a c) \geq \angle(\tilde{b} \tilde{a} \tilde{c})$ and $\angle(a b c) \geq \angle(\tilde{a} \tilde{b} \tilde{c})$ because the angle at $\tilde{u}$ of a quadrangle $\tilde{a} \tilde{u} \tilde{b} \tilde{c}$ is less than or equal to $\pi$ where the quadrangle $\tilde{a} \tilde{u} \tilde{b} \tilde{c}$ consists of comparison triangle domains $\triangle(\tilde{a} \tilde{u} \tilde{c})$ and $\triangle(\tilde{b} \tilde{u} \tilde{c})$. In the same way we can prove that $\angle(a c b) \geq \angle(\tilde{a} \tilde{c} \tilde{b})$. Therefore, we have proved that the condition (C) is satisfied in $U(w)$.

It remains to treat the case that $w$ is a vertex of $\triangle(p q r)$. We have only to consider a geodesic triangle domain $\triangle(a b c)$ containing $w$. In order to prove that $\angle(a b c) \geq \angle(\tilde{a} \tilde{b} \tilde{c})$, we first show that there exists a point $x \in T(b, c)$ such that a minimal geodesic segment $T(a, x)$ passes through $w$ or a geodesic biangle domain $V(a, x)$ contains $w$ where $V(a, x)$ is surrounded by two minimal geodesic segments connecting $a$ and $x$. If one of geodesic biangles $V(a, b)$ and $V(a, c)$ exists and contains $w$, then $x=b$ or $c$ satisfies the condition. Otherwise, since $w$ lies in one side of $T(a, y)$ in $\triangle(a b c)$ if $y \in T(b, c)$ is near $b$, and in the different side of $T(a, y)$ if $y \in T(b, c)$ is near $c$, we can find a point $x \in T(b, c) \backslash\{b, c\}$ satisfying the above condition.

Using this point $x$, we consider the quadrangle $\tilde{a} \tilde{b} \tilde{x} \tilde{c}$ consisting of comparison triangle domains $\triangle(\tilde{a} \tilde{b} \tilde{x})$ and $\triangle(\tilde{a} \tilde{x} \tilde{c})$. Using the hinge comparison theorem in Lemma 3 (H) to make 
the comparison triangle domain $\triangle(\tilde{a} \tilde{b} \tilde{c})$ from the quadrangle $\tilde{a} \tilde{b} \tilde{x} \tilde{c}$, we have

$$
\angle(a b c) \geq \angle(\tilde{a} \tilde{b} \tilde{x}) \geq \angle(\tilde{a} \tilde{b} \tilde{c})
$$

The other cases are proved in the same way. Thus Lemma $3(\mathrm{C})$ is satisfied in some neighborhood around $w$.

The map $\varphi: D \rightarrow \bar{D}$ is given by $\varphi(x)=x$ for any point $x \in D \backslash \triangle(p q r)$ and by $\varphi(x)=\varphi_{1}(x)$ for any $x \in \Delta(p q r)$ where $\varphi_{1}: \Delta(p q r) \rightarrow \widetilde{\triangle}(p q r)$ is the compression map given in Lemma 4.

As a special case we prove the following lemma.

LEMMA 6. Let D be a polygonal surface with curvature bounded below by $\kappa$ and let $V(p, q)$ be a geodesic biangle domain with vertices $p$ and $q$ in $D$, namely $V(p, q)$ is a domain in $D$ surrounded by two minimal geodesic segments $T_{1}(p, q)$ and $T_{2}(p, q)$ connecting $p$ and $q$. Let $\bar{D}$ be the surface constructed as follows. The biangle domain $V(p, q)$ is removed from $D$ and then the boundary of $D \backslash V(p, q), T_{1}(p, q)$ and $T_{2}(p, q)$, are glued together along corresponding points. Assume that the distances between the adjacent vertices in $\partial \bar{D}$ do not change. Then, $\bar{D}$ is also a polygonal surface with curvature bounded below by $\kappa$. There exists a compression map $\varphi: D \rightarrow \bar{D}$ such that the restriction to each side is an isometry to the corresponding side and $d(\varphi(x), \varphi(y)) \leq d(x, y)$ for any points $x, y \in D$.

If $p$ and $q$ are adjacent vertices in $\partial D$, then $\bar{D}$ satisfies the assumption. If there exists a diagonal $T(p, q)$ of $D$ used in a minimum spanning tree $\operatorname{MST}(D)$ such that $V(p, q)$ is contained in a sufficiently thin neighborhood around $T(p, q)$, then Lemma 9 shows that $\bar{D}$ satisfies the assumption. This remark, together with the remark after the statement of Lemma 5 , will play important roles in the deformation of polygonal surfaces which is discussed in Section 4 and also in the proof of Lemma 23.

Proof. This lemma is proved in the same way as Lemma 5. Let $T(p, q)$ be the minimal geodesic segment in $\bar{D}$ reduced from $V(p, q)$. Depending on $w \notin T(p, q), w \in \operatorname{Int} T(p, q)$ or $w \in\{p, q\}$, we find a neighborhood $U(w)$ satisfying the condition (C) in Lemma 3 .

The map $\varphi: D \rightarrow \bar{D}$ is given by $\varphi(x)=x$ for any $x \in D \backslash V(p, q)$ and $\varphi(x)=\varphi_{2}(x)$ for any $x \in V(p, q)$ where $\varphi_{2}: V(p, q) \rightarrow T_{1}(p, q)$ is the map in (2) of Lemma 4.

3. Steiner trees and polygonal surfaces. In this section we summarize some properties of Steiner minimum trees in a complete Alexandrov surface and a polygonal surface with curvature bounded below by $\kappa$.

PROPOSITION 7. Let $M$ be a complete Alexandrov surface with curvature bounded below by $\kappa$ and $P$ a set of $n$ points in $M$. Then, a Steiner minimum tree $\operatorname{SMT}_{M}(P)$ for $P$ in $M$ satisfies the following properties.

(1) All terminal points of $\mathrm{SMT}_{M}(P)$ are points in $P$.

(2) Any two edges meet at an angle at least $2 \pi / 3$. 
(3) Every Steiner point has degree exactly three, and hence is not a singular point in $M$. The edges emanating from it to three neighboring vertices are unique minimal geodesic segments.

(4) There are at most $n-2$ Steiner points in $\operatorname{SMT}_{M}(P)$.

PROOF. The properties (1) and (4) are proved in the same way as in the Euclidean plane $\boldsymbol{E}^{2}$. We prove (2). Suppose that there exists a vertex $p$ where two edges meet at the angle $\theta$ less than $2 \pi / 3$. Let $T(p, q)$ and $T(p, r)$ be those edges. Let $q_{1}$ and $r_{1}$ be points in $T(p, q)$ and $T(p, r)$ with $a=d\left(p, q_{1}\right)=d\left(p, r_{1}\right)$. For a sufficiently small positive $\varepsilon$, the subarc $C(p, \varepsilon)=\{y \in M ; d(p, y)=\varepsilon\} \cap \Delta\left(p q_{1} r_{1}\right)$ of the circle contains a point $s$ with $b(\varepsilon)=d\left(s, q_{1}\right)=d\left(s, r_{1}\right)$ since $d\left(r_{1}, q_{2}\right)>d\left(r_{1}, r_{2}\right)$ and $d\left(q_{1}, r_{2}\right)>d\left(q_{1}, q_{2}\right)$, where $q_{2}$ and $r_{2}$ are the endpoints of the subarc of $C(p, \varepsilon)$ in $T\left(p, q_{1}\right)$ and $T\left(p, r_{1}\right)$, respectively. The quadrangle $\tilde{p} \tilde{q}_{1} \tilde{s} \tilde{r}_{1}$ consisting of comparison triangle domains $\triangle\left(\tilde{p} \tilde{q}_{1} \tilde{s}\right)$ and $\triangle\left(\tilde{p} \tilde{r}_{1} \tilde{s}\right)$ has the angle $L\left(\tilde{q}_{1} \tilde{p} \tilde{r}_{1}\right)$ less than $2 \pi / 3$. Set $\theta_{1}=L\left(\tilde{q}_{1} \tilde{p} \tilde{s}\right)=L\left(\tilde{r}_{1} \tilde{p} \tilde{s}\right) \leq \theta / 2<\pi / 3$. We consider the hinge $(a, \varepsilon, \theta / 2)$ in $M(\kappa)$. If the triangle constructed by this hinge is $\triangle(\tilde{q} 1 \tilde{p} \tilde{x}(\varepsilon))$, we have $a-\varepsilon / 2>d\left(\tilde{q}_{1}, \tilde{x}(\varepsilon)\right)$ for any sufficiently small positive $\varepsilon$, using the first variation formula for the geodesic variation through $T\left(\tilde{q}_{1}, \tilde{p}\right)$ in $M(\kappa)$ with fixed point $q_{1}$. Therefore, we have

$$
a-\frac{\varepsilon}{2}>d\left(\tilde{q}_{1}, \tilde{x}(\varepsilon)\right) \geq d\left(\tilde{q}_{1}, \tilde{s}\right)=d\left(q_{1}, s\right)=b(\varepsilon),
$$

because the middle inequality follows from $\theta_{1} \leq \theta / 2$. For those $\varepsilon$, we have

$$
\begin{aligned}
d\left(p, q_{1}\right)+d\left(p, r_{1}\right) & =d\left(\tilde{p}, \tilde{q}_{1}\right)+d\left(\tilde{p}, \tilde{r}_{1}\right) \\
& =2 a \\
& >2\left(b(\varepsilon)+\frac{\varepsilon}{2}\right) \\
& =d\left(\tilde{q}_{1}, \tilde{s}\right)+d\left(\tilde{r}_{1}, \tilde{s}\right)+d(\tilde{p}, \tilde{s}) \\
& =d\left(q_{1}, s\right)+d\left(r_{1}, s\right)+d(p, s),
\end{aligned}
$$

contradicting that $T(p, q)$ and $T(p, r)$ are edges in $\operatorname{SMT}_{M}(P)$.

The property ( 3 ) follows from (2) because there are at least three edges emanating from this point, each angle is at least $2 \pi / 3$ and the total angle around this point is at most $2 \pi$.

We say that a tree $T$ is a Steiner tree if $T$ satisfies (1) to (3). A Steiner tree $T$ is by definition full if $T$ has exactly $n-2$ Steiner points. Any Steiner tree can be decomposed into an edge-disjoint union of full Steiner trees.

We consider the minimum network problem in a polygonal surface $D$ with curvature bounded below by $\kappa$ and vertex set $v(\partial D)$. A Steiner minimum tree for $v(\partial D)$ in $D$ and a minimum spanning tree on $v(\partial D)$ in $D$ are denoted by $\operatorname{SMT}(D)$ and $\operatorname{MST}(D)$, respectively. We say that an oriented curve $C$ in $D$ is right convex (resp. left convex) if for any point $w$ in $C$ there exists a positive $\varepsilon$ such that any minimal geodesic segment $T(u, v)$ connecting any points $u, v \in C$ contained in the $\varepsilon$-ball with center $w$ is in the right (resp. left ) side of $C$ or on $C$. 
PROPOSITION 8. Let $D$ be a polygonal surface with curvature bounded below by $\kappa$ and vertex set $v(\partial D)$. Then, $\mathrm{SMT}(D)$ satisfies the following properties.

(1) All terminal points of $\operatorname{SMT}(D)$ are points in $v(\partial D)$. The interior of every edge of $\operatorname{SMT}(D)$ does not intersect $\partial D$ if it is not an edge of $\partial D$.

(2) Any two edges meet at an angle of at least $2 \pi / 3$. Hence, if a vertex of $\operatorname{SMT}(D)$ is not a spiral vertex of $\partial D$, then the vertex has degree at most 3 .

(3) All Steiner points are in Int $D$ and have degree exactly three. Hence, they are not singular points in Int $D$. The edges emanating from them to three neighboring vertices are unique minimal geodesic segments.

(4) If $v(\partial D)$ consists of $n$ points, then there are at most $n-2$ Steiner points in $\operatorname{SMT}(D)$.

(5) Assume that $\operatorname{SMT}(D)$ is full. Then, the vertices $p$ and $q \in v(\partial D)$ are adjacent in $\partial D$ if and only if the minimal subtree $S(p, q)$ of $\operatorname{SMT}(D)$ is convex.

PROOF. We omit the proofs of (1) to (4). We prove (5). Since SMT $(D)$ is full, any edge in $\partial D$ is not contained in $\operatorname{SMT}(D)$. Suppose for indirect proof that $S(p, q)$ is right convex and $q$ is not the adjacent point of $p$ in $\partial D$. Take a vertex $r \in P$ lying in the subarc of $\partial D$ from $p$ to $q$. Consider a subtree $S(r, p)$ of $\operatorname{SMT}(D)$ from $r$ to $p$. Then, $S(p, q)$ is not right convex at the first intersection of $S(r, p)$ and $S(p, q)$ which is a Steiner point, a contradiction.

Conversely, if $S(p, q)$ is not convex, there exists a convex subtree $S$ such that the first two edges coincide with those of $S(p, q)$. This implies that $S$ connects $p$ and a vertex between $p$ and $q$ in $\partial D$. Thus, $p$ and $q$ are not adjacent in $\partial D$.

We recall that a shortest network interconnecting the vertex set $v(\partial D)$ in a polygonal surface $D$ without using any vertices other than $v(\partial D)$ is a minimum spanning tree on $v(\partial D)$ in $D$. Let $\operatorname{mst}(D)$ be the set of all topologies of all minimum spanning trees on $v(\partial D)$ in $D$. A minimum spanning tree with topology $s \in \operatorname{mst}(D)$ is denoted by $r(s, D)$. Therefore, $r(s, D)$ is one of $\operatorname{MST}(D)$ 's. In general, $r(s, D)$ is not unique for $s \in \operatorname{mst}(D)$. Du and Hwang [6] call $r(s, D)$ a minimum inner spanning tree with topology $s$ when $D=C(T)$ is a characteristic area for a full Steiner tree $T$ in $\boldsymbol{E}^{2}$.

We summarize some properties of $\operatorname{mst}(D)$.

Lemma 9. Let $D$ be a polygonal surface with curvature bounded below by $\kappa$ and vertex set $v(\partial D)$. The following are true.

(1) $\operatorname{mst}(D)$ is a finite set.

(2) $L(r(s, D))$ is constant for any $s \in \operatorname{mst}(D)$.

(3) All edges of $r(s, D)$ do not cross any other edge of $r\left(s^{\prime}, D\right)$ at their interior point for any $s, s^{\prime} \in \operatorname{mst}(D)$.

PROOF. We omit the proofs of (1) and (2). The property (3) is proved by Rubinstein and Thomas [14]. Since this property is important in our deformation of polygonal surfaces in Section 4, we refer to the proof here. They reduced the number of cases, however we do not in the following proof. Suppose for indirect proof that edges $T_{1}=T(a, b) \subset r_{1}=r(s, D)$ and $T_{2}=T(c, d) \subset r_{2}=r\left(s^{\prime}, D\right)$ with $T_{1} \neq T_{2}$ intersect at their interior point $p \in \operatorname{Int} T_{1} \cap \operatorname{Int} T_{2}$. 
Then, $T_{1} \cap T_{2}=\{p\}$ and both $r_{1} \backslash \operatorname{Int} T_{1}$ and $r_{2} \backslash$ Int $T_{2}$ consists of two connected components. Set $T_{1}(a), T_{1}(b), T_{2}(c)$ and $T_{2}(d)$ are those connected components containing $a, b, c$ and $d$, respectively. The following cases happen.

(1) (a) $T_{1}(a) \ni c, T_{1}(b) \ni d$; (b) $T_{1}(a) \ni d, T_{1}(b) \ni c$.

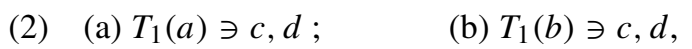

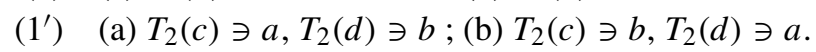

$\begin{array}{lll}\left(2^{\prime}\right) & \text { (a) } T_{2}(c) \ni a, b ; & \text { (b) } T_{2}(d) \ni a, b .\end{array}$

We may suppose without loss of generality that $d(a, b) \leq d(c, d)$. Since $T_{1} \neq T_{2}$, we have

$$
\begin{aligned}
d(a, b)+d(c, d) & =d(a, p)+d(p, b)+d(c, p)+d(p, d) \\
& >d(a, d)+d(b, c)
\end{aligned}
$$

and

$$
\begin{aligned}
d(a, b)+d(c, d) & =d(a, p)+d(p, b)+d(c, p)+d(p, d) \\
& >d(a, c)+d(b, d) .
\end{aligned}
$$

Therefore, we have $d(c, d)>\min \{d(a, d), d(b, c)\}$ and $d(c, d)>\min \{d(a, c), d(b, d)\}$. Each of $\left(1^{\prime}\right)\left(\right.$ a) and $\left(1^{\prime}\right)(b)$, say $\left(1^{\prime}\right)($ a), causes a contradiction as follows. Notice that both $S_{1}=T_{2}(c) \cup T(a, d) \cup T_{2}(d)$ and $S_{2}=T_{2}(c) \cup T(b, c) \cup T_{2}(d)$ are spanning trees in $D$. Then, we have

$$
\begin{aligned}
L(\operatorname{MST}(D)) & =L\left(r_{2}\right) \\
& =L\left(T_{2}(c)\right)+L(T(c, d))+L\left(T_{2}(d)\right) \\
& >L\left(T_{2}(c)\right)+\min \{d(a, d), d(b, c)\}+L\left(T_{2}(d)\right) \\
& =\min \left\{L\left(S_{1}\right), L\left(S_{2}\right)\right\} .
\end{aligned}
$$

Suppose that (1) (a) and ( $2^{\prime}$ ) (a) happen. Consider two spanning trees $S_{1}=T_{1}(a) \cup$ $T(b, c) \cup T_{1}(b)$ and $S_{2}=T_{2}(c) \cup T(a, d) \cup T_{2}(d)$ in $D$. Then, we have

$$
\begin{aligned}
L(\operatorname{MST}(D)) & =L\left(r_{1}\right) \\
& =L\left(T_{1}(a)\right)+L(T(a, b))+L\left(T_{1}(b)\right),
\end{aligned}
$$

and

$$
\begin{aligned}
L(\operatorname{MST}(D)) & =L\left(r_{2}\right) \\
& =L\left(T_{2}(c)\right)+L(T(c, d))+L\left(T_{2}(d)\right) .
\end{aligned}
$$

Therefore, we have

$$
\begin{aligned}
2 L & (\operatorname{MST}(D))=L\left(r_{1}\right)+L\left(r_{2}\right) \\
& =L\left(T_{1}(a)\right)+L(T(a, b))+L\left(T_{1}(b)\right)+L\left(T_{2}(c)\right)+L(T(c, d))+L\left(T_{2}(d)\right) \\
& >L\left(T_{1}(a)\right)+L(T(b, c))+L\left(T_{1}(b)\right)+L\left(T_{2}(c)\right)+L(T(a, d))+L\left(T_{2}(d)\right) \\
& =L\left(S_{1}\right)+L\left(S_{2}\right) .
\end{aligned}
$$


Thus one of $L\left(S_{1}\right)$ and $L\left(S_{2}\right)$ is less than $L(\operatorname{MST}(D))$, a contradiction. In the other cases, the same computation causes a contradiction. The following is the list of spanning trees in $D$ we consider in the remaining cases.

1. Case; (1) (a) and (2') (b): $T_{1}(a) \cup T(a, d) \cup T_{1}(b)$ and $T_{2}(c) \cup T(b, c) \cup T_{2}(d)$.

2. Case; (1) (b) and (2') (a): $T_{1}(a) \cup T(a, c) \cup T_{1}(b)$ and $T_{2}(c) \cup T(b, d) \cup T_{2}(d)$.

3. Case; (1) (b) and (2') (b): $T_{1}(a) \cup T(b, d) \cup T_{1}(b)$ and $T_{2}(c) \cup T(a, c) \cup T_{2}(d)$.

4. Case; (2) (a) and (2') (a): $T_{1}(a) \cup T(b, c) \cup T_{1}(b)$ and $T_{2}(c) \cup T(a, d) \cup T_{2}(d)$.

5. Case; (2) (a) and (2') (b): $T_{1}(a) \cup T(b, d) \cup T_{1}(b)$ and $T_{2}(c) \cup T(a, c) \cup T_{2}(d)$.

6. Case; (2) (b) and (2') (a): $T_{1}(a) \cup T(a, c) \cup T_{1}(b)$ and $T_{2}(c) \cup T(b, d) \cup T_{2}(d)$.

7. Case; (2) (b) and (2') (b): $T_{1}(a) \cup T(a, d) \cup T_{1}(b)$ and $T_{2}(c) \cup T(b, c) \cup T_{2}(d)$.

In Lemma 10, we make arrangements to start the deformation of the polygonal surface $D$ in Theorem 1. This arrangements ensures that the resulting polygonal surface $D_{0}$ does not degenerate into a metric space with lower dimension under the deformation in Section 4.

Let $\Gamma(D)$ denote the union of all edges in all minimum spanning trees in $D$, namaly, $\Gamma(D)=\bigcup_{s \in \operatorname{mst}(D)} r(s, D)$.

LEMMA 10. Let $D$ be a polygonal surface with curvature bounded below by $\kappa$ and vertex set $v(\partial D)$. Then, there exists a polygonal surface $D_{0}$ with curvature bounded below by $\kappa$ and vertex set $v\left(\partial D_{0}\right)$ satisfying the following.

(1) If all $p_{i}^{\prime} \in v\left(\partial D_{0}\right)$ correspond to $p_{i} \in v(\partial D), i=1,2, \ldots, n$, in this order on $\partial D_{0}$ and $\partial D$, then $d\left(p_{i}^{\prime}, p_{i+1}^{\prime}\right) \leq d\left(p_{i}, p_{i+1}\right)$ where $p_{n+1}^{\prime}=p_{1}^{\prime}$ and $p_{n+1}=p_{1}$.

(2) $\operatorname{mst}(D) \subset \operatorname{mst}\left(D_{0}\right)$ and $\partial D_{0} \subset \Gamma\left(D_{0}\right)$.

(3) $L\left(\operatorname{MST}\left(D_{0}\right)\right)=L(\operatorname{MST}(D))$ and $L\left(\operatorname{SMT}\left(D_{0}\right)\right) \leq L(\operatorname{SMT}(D))$.

Proof. If $\partial D \subset \Gamma(D)$, then $D_{0}=D$ is a polygonal surface satisfying the conditions. Suppose that there exists an edge $T=T\left(p_{i}, p_{i+1}\right) \subset \partial D$ such that $T \not \subset \Gamma(D)$. From Lemma 9 (3), $\Gamma(D) \cup \partial D$ divides $D$ into a finitely many simply connected domains, namely $D \backslash(\Gamma(D) \cup \partial D)$ consists of simply connected open domains. One of their closures contains $T$, say $D^{\prime}$. From the construction, $\partial D^{\prime} \backslash T \subset \Gamma(D)$, and hence $T$ is the longest in all the edges in $\partial D^{\prime}$. Let $q$ be the midpoint of $T$. Let $\widetilde{\triangle}(s)$ be the triangle in $M(\kappa)$ determined by the lengths of three sides, $L(T) / 2, L(T) / 2$ and $L(T)-s$, for any positive $s \in(0, L(T))$. Glue the triangle $\widetilde{\triangle}(s)$ on $D^{\prime}$ along $T\left(p_{i}, q\right) \cup T\left(q, p_{i+1}\right)$. The resulting domain is denoted by $D^{\prime}(s)$. The new edge which connects $p_{i}$ and $p_{i+1}$ is denoted by $T(s)$. Let $\iota: D^{\prime} \rightarrow D^{\prime}(s)$ be the inclusion map which is not isometric. Then, there exists a positive $s_{1}$ such that $\Gamma\left(D^{\prime}(s)\right)=\iota\left(\Gamma\left(D^{\prime}\right)\right)$ for all $s<s_{1}$ and $\iota\left(\Gamma\left(D^{\prime}\right)\right) \subsetneq \Gamma\left(D^{\prime}\left(s_{1}\right)\right)$. If $T\left(s_{1}\right) \subset \Gamma\left(D^{\prime}\left(s_{1}\right)\right)$, then $D^{\prime}$ is replaced by $D^{\prime}\left(s_{1}\right)$ in $D$. Otherwise, repeat this construction by using $T\left(s_{1}\right)$, its midpoint, and the suitable triangles in $M(\kappa)$ instead of $T, q$ and $\widetilde{\triangle}(s)$ until the new edge is contained in the union of MST's. This process will stop after a finite number of iterations because of Lemma 9 (3). The resulting domain is replaced by $D^{\prime}$ in $D$ and we have a polygonal surface $D_{1}$ with curvature bounded below by $\kappa$, as was seen in the proof of Lemma 5 . Since $D$ is included in $D_{1}$ as a subset by the inclusion map $\iota$ and the lengths of curves $C$ in $D$ are equal to those of curves 
$\iota(C)$ in $D_{1}$, we have $L(\operatorname{SMT}(D)) \geq L\left(\operatorname{SMT}\left(D_{1}\right)\right)$. If $\partial D_{1} \not \subset \Gamma\left(D_{1}\right)$, namely, there exists an edge $T^{\prime} \subset \partial D$ other than $T$ with $T^{\prime} \not \subset \Gamma(D)$, we repeat this deformation until we have a polygonal surface $D_{0}$ such that $\partial D_{0} \subset \Gamma\left(D_{0}\right)$

4. Deformation of polygonal surfaces. Let $D_{0}$ be a polygonal surface with curvature bounded below by $\kappa$ and $v\left(\partial D_{0}\right)$ the vertex set of $\partial D_{0}$. Assume that $\partial D_{0} \subset \Gamma\left(D_{0}\right)$. Such a polygonal surface $D_{0}$ has been constructed in Lemma 10. Let $\mathcal{M}$ be the set of all polygonal surfaces $D_{\alpha}, \alpha \in \Lambda$, with curvature bounded below by $\kappa$ satisfying the following conditions.

(P1) Every vertex in the boundary $\partial D_{\alpha}$ corresponds to a point in $v\left(\partial D_{0}\right)$ and is denoted by the same symbol. Namely, $v\left(\partial D_{\alpha}\right)=v\left(\partial D_{0}\right)$.

(P2) There exists a compression map $\varphi_{\alpha}: D_{0} \rightarrow D_{\alpha}$. For convenience, we think that $\partial D_{0}=\partial D_{\alpha}=\varphi_{\alpha}\left(\partial D_{0}\right)$.

(P3) $\operatorname{mst}\left(D_{\alpha}\right) \supset \operatorname{mst}\left(D_{0}\right)$.

(P4) $L\left(r\left(s, D_{\alpha}\right)\right)=L\left(r\left(s, D_{0}\right)\right)$ for any $s \in \operatorname{mst}\left(D_{0}\right)$ where $r\left(s, D_{0}\right)$ and $r\left(s, D_{\alpha}\right)$ are by definition the minimum spanning trees on $v\left(\partial D_{0}\right)$ in $D_{0}$ and $D_{\alpha}$ with topology $s$, respectively.

(P5) $\partial D_{\alpha} \subset \Gamma\left(D_{\alpha}\right)$ where $\Gamma\left(D_{\alpha}\right)$ is the union of all minimum spanning trees on $v\left(\partial D_{\alpha}\right)$ in $D_{\alpha}$.

We define a binary relation $\leq$ in $\mathcal{M}$ as follows. The relation $D_{\alpha} \leq D_{\beta}$ holds for $D_{\alpha}, D_{\beta} \in \mathcal{M}$ if there exists a compression map $\varphi_{\alpha \beta}: D_{\alpha} \rightarrow D_{\beta}$ with $\varphi_{\beta}=\varphi_{\alpha \beta} \circ \varphi_{\alpha}$ and $\operatorname{mst}\left(D_{\alpha}\right) \subset \operatorname{mst}\left(D_{\beta}\right)$. From Lemmas 5, 6 and the remarks after those lemmas, we can make $D_{\beta}$ and $\varphi_{\alpha \beta}$ if there exists a point $p \in D_{\alpha}$ with $p \notin \Gamma\left(D_{\alpha}\right)$ such that any neighborhood of $p$ is not isometric to any domain in $M(\kappa)$. We can prove the following, and therefore $\leq$ is a partial order in $\mathcal{M}$.

LEMMA 11. The relation $\leq$ satisfies the following.

(1) $D_{\alpha} \leq D_{\alpha}$ for all $\alpha \in \Lambda$.

(2) If $D_{\alpha} \leq D_{\beta}$ and $D_{\beta} \leq D_{\gamma}$, then $D_{\alpha} \leq D_{\gamma}$.

(3) If $D_{\alpha} \leq D_{\beta}$ and $D_{\beta} \leq D_{\alpha}$, then $D_{\alpha}=D_{\beta}$, namely, $\varphi_{\alpha \beta}$ is an isometry from $D_{\alpha}$ onto $D_{\beta}$.

Proof. (1) and (2) are direct consequences from the definition. We prove (3). Since $\varphi_{\alpha}=\varphi_{\beta \alpha} \circ \varphi_{\beta}$ and $\varphi_{\beta}=\varphi_{\alpha \beta} \circ \varphi_{\alpha}$, we have

$$
\varphi_{\alpha}=\left(\varphi_{\beta \alpha} \circ \varphi_{\alpha \beta}\right) \circ \varphi_{\alpha} \quad \text { and } \quad \varphi_{\beta}=\left(\varphi_{\alpha \beta} \circ \varphi_{\beta \alpha}\right) \circ \varphi_{\beta} .
$$

Therefore, $\varphi_{\beta \alpha} \circ \varphi_{\alpha \beta}$ and $\varphi_{\alpha \beta} \circ \varphi_{\beta \alpha}$ are identity maps because $\varphi_{\alpha}$ and $\varphi_{\beta}$ are surjective. Hence, we have

$$
\begin{aligned}
d(x, y) & =d\left(\varphi_{\beta \alpha} \circ \varphi_{\alpha \beta}(x), \varphi_{\beta \alpha} \circ \varphi_{\alpha \beta}(y)\right) \\
& \leq d\left(\varphi_{\alpha \beta}(x), \varphi_{\alpha \beta}(y)\right) \leq d(x, y)
\end{aligned}
$$

for any $x, y \in D_{\alpha}$. Therefore, $\varphi_{\alpha \beta}: D_{\alpha} \rightarrow D_{\beta}$ is an isometry. In the same way we have that $\varphi_{\beta \alpha}: D_{\beta} \rightarrow D_{\alpha}$ is an isometry. 
Let $\operatorname{SMT}\left(D_{\alpha}\right)$ and $\operatorname{MST}\left(D_{\alpha}\right)$ be a Steiner minimum tree for $v\left(\partial D_{\alpha}\right)$ in $D_{\alpha}$ and a minimum spanning tree on $v\left(\partial D_{\alpha}\right)$ in $D_{\alpha}$, respectively.

LEMMA 12. The following are true.

(1) $L\left(\operatorname{MST}\left(D_{\alpha}\right)\right)=L\left(\operatorname{MST}\left(D_{0}\right)\right)$ for any $D_{\alpha} \in \mathcal{M}$.

(2) $L\left(\operatorname{SMT}\left(D_{\alpha}\right)\right) \geq L\left(\operatorname{SMT}\left(D_{\beta}\right)\right)$ for any $D_{\alpha}, D_{\beta} \in \mathcal{M}$ with $D_{\alpha} \leq D_{\beta}$.

Proof. Let $s \in \operatorname{mst}\left(D_{0}\right)$. Then, $s \in \operatorname{mst}\left(D_{\alpha}\right)$. Therefore, we have

$$
L\left(\operatorname{MST}\left(D_{\alpha}\right)\right)=L\left(r\left(s, D_{\alpha}\right)\right)=L\left(r\left(s, D_{0}\right)\right)=L\left(\operatorname{MST}\left(D_{0}\right)\right)
$$

because of the condition (P4). This proves (1). If $D_{\alpha} \leq D_{\beta}$ for $\alpha, \beta \in \Lambda$, then we have

$$
L\left(\operatorname{SMT}\left(D_{\alpha}\right)\right) \geq L\left(\varphi_{\alpha \beta}\left(\operatorname{SMT}\left(D_{\alpha}\right)\right)\right) \geq L\left(\operatorname{SMT}\left(D_{\beta}\right)\right) .
$$

This proves (2).

We also define the partial order in the index set $\Lambda$, namely $\alpha \leq \beta$ for $\alpha, \beta \in \Lambda$ if and only if $D_{\alpha} \leq D_{\beta}$. We will prove that $(\mathcal{M}, \leq)$ is an inductively ordered set. Let $\mathcal{M}_{0}$ be a totally ordered subset of $\mathcal{M}$ with index set $\Lambda_{0} \subset \Lambda$. If $\Lambda_{0}$ is a finite set, we then find an $\alpha_{0} \in \Lambda_{0}$ such that $D_{\alpha} \leq D_{\alpha_{0}}$ for all $\alpha \in \Lambda_{0}$. Here $D_{\alpha_{0}} \in \mathcal{M}_{0}$ is an upper bound of $\mathcal{M}_{0}$. Thus we may assume that $\Lambda_{0}$ is not a finite set.

We will construct an upper bound $N$ for $\mathcal{M}_{0}$. We define an equivalence relation $\sim$ in $D_{0}$ by

$$
x \sim y \quad \text { if } \quad \inf _{\alpha \in \Lambda_{0}} d\left(\varphi_{\alpha}(x), \varphi_{\alpha}(y)\right)=0 .
$$

Let $\bar{x}$ be the equivalence class containing $x \in D_{0}$ and $N=D_{0} / \sim$. We define a distance in $N$ as follows.

$$
d(\bar{x}, \bar{y})=\inf _{\alpha \in \Lambda_{0}} d\left(\varphi_{\alpha}(x), \varphi_{\alpha}(y)\right)
$$

for any $\bar{x}, \bar{y} \in N$. This distance is well defined in $N$ and satisfies that

$$
d(\bar{x}, \bar{y}) \leq d\left(\varphi_{\beta}(x), \varphi_{\beta}(y)\right) \leq d\left(\varphi_{\alpha}(x), \varphi_{\alpha}(y)\right) \leq d(x, y)
$$

for any $x, y \in D_{0}$ and $\alpha, \beta \in \Lambda_{0}$ with $\alpha \leq \beta$. In the same way we define metric spaces $N_{\alpha}=D_{0} / \sim_{\alpha}$ by using equivalence relations $\sim_{\alpha}$ in $D_{0}$ where $x \sim_{\alpha} y$ for $x, y \in D_{0}$ if $d\left(\varphi_{\alpha}(x), \varphi_{\alpha}(y)\right)=0$. Let $[x]_{\alpha}$ be the equivalence class containing $x \in D_{0}$. We define the distance $d_{\alpha}$ by $d_{\alpha}\left([x]_{\alpha},[y]_{\alpha}\right)=d\left(\varphi_{\alpha}(x), \varphi_{\alpha}(y)\right)$ for any $[x]_{\alpha},[y]_{\alpha} \in N_{\alpha}$. Then, the map $\bar{\varphi}_{\alpha}: N_{\alpha} \rightarrow D_{\alpha}$ given by $\bar{\varphi}_{\alpha}\left([x]_{\alpha}\right)=\varphi_{\alpha}(x)$ for any $[x]_{\alpha} \in N_{\alpha}$ is isometric. The sequence of metric spaces $\left(N_{\alpha}, d_{\alpha}\right)$ converges to $(N, d)$ in some sense.

Let $f_{\alpha}: D_{0} \times D_{0} \rightarrow \boldsymbol{R}$ be a function given by $f_{\alpha}(x, y)=d_{\alpha}\left([x]_{\alpha},[y]_{\alpha}\right)-d(\bar{x}, \bar{y})$ for any $[x]_{\alpha},[y]_{\alpha} \in N_{\alpha}$, where $\alpha \in \Lambda_{0}$. In other words, $f_{\alpha}(x, y)=d\left(\varphi_{\alpha}(x), \varphi_{\alpha}(y)\right)-d(\bar{x}, \bar{y})$ for any $x, y \in D_{0}$.

LEMMA 13. The function $f_{\alpha}$ is nonnegative. Given $x, y \in D_{0}, f_{\alpha}(x, y)$ is monotone nonincreasing for $\alpha \in \Lambda_{0}$. For any $\varepsilon>0$ there exists an $\alpha_{0} \in \Lambda_{0}$ such that $f_{\alpha}(x, y)<\varepsilon$ for any $x, y \in D_{0}$ and any $\alpha \in \Lambda_{0}$ with $\alpha \geq \alpha_{0}$. 
PROOF. We have already proved the first part of the lemma.

Let $m_{\alpha}=\max \left\{f_{\alpha}(x, y) ; x, y \in D_{0}\right\}$. Let $x_{\alpha}$ and $y_{\alpha}$ be points in $D_{0}$ such that $f_{\alpha}\left(x_{\alpha}, y_{\alpha}\right)=m_{\alpha}$. Since $D_{0}$ is compact, there exist subsequences $\left\{x_{\alpha_{j}}\right\}$ and $\left\{y_{\alpha_{j}}\right\}$ of $\left\{x_{\alpha}\right\}$ and $\left\{y_{\alpha}\right\}$ which converge to some points $x_{0}$ and $y_{0}$, respectively. Since

$$
\begin{aligned}
m_{\alpha_{j}}= & d\left(\varphi_{\alpha_{j}}\left(x_{\alpha_{j}}\right), \varphi_{\alpha_{j}}\left(y_{\alpha_{j}}\right)\right)-d\left(\bar{x}_{\alpha_{j}}, \bar{y}_{\alpha_{j}}\right) \\
\leq & d\left(\varphi_{\alpha_{j}}\left(x_{\alpha_{j}}\right), \varphi_{\alpha_{j}}\left(x_{0}\right)\right)+d\left(\varphi_{\alpha_{j}}\left(x_{0}\right), \varphi_{\alpha_{j}}\left(y_{0}\right)\right) \\
& +d\left(\varphi_{\alpha_{j}}\left(y_{0}\right), \varphi_{\alpha_{j}}\left(y_{\alpha_{j}}\right)\right)-d\left(\bar{x}_{\alpha_{j}}, \bar{y}_{\alpha_{j}}\right) \\
\leq & d\left(x_{\alpha_{j}}, x_{0}\right)+d\left(\varphi_{\alpha_{j}}\left(x_{0}\right), \varphi_{\alpha_{j}}\left(y_{0}\right)\right)+d\left(y_{0}, y_{\alpha_{j}}\right)-d\left(\bar{x}_{\alpha_{j}}, \bar{y}_{\alpha_{j}}\right),
\end{aligned}
$$

and

$$
\begin{aligned}
d\left(\bar{x}_{0}, \bar{y}_{0}\right) & \leq d\left(\bar{x}_{0}, \bar{x}_{\alpha_{j}}\right)+d\left(\bar{x}_{\alpha_{j}}, \bar{y}_{\alpha_{j}}\right)+d\left(\bar{y}_{\alpha_{j}}, \bar{y}_{0}\right) \\
& \leq d\left(x_{0}, x_{\alpha_{j}}\right)+d\left(\bar{x}_{\alpha_{j}}, \bar{y}_{\alpha_{j}}\right)+d\left(y_{\alpha_{j}}, y_{0}\right),
\end{aligned}
$$

we have that

$$
m_{\alpha_{j}} \leq d\left(\varphi_{\alpha_{j}}\left(x_{0}\right), \varphi_{\alpha_{j}}\left(y_{0}\right)\right)-d\left(\bar{x}_{0}, \bar{y}_{0}\right)+2 d\left(x_{0}, x_{\alpha_{j}}\right)+2 d\left(y_{0}, y_{\alpha_{j}}\right) .
$$

Thus, the sequence $\left\{m_{\alpha_{j}}\right\}$ converges to 0 as $j$ goes to $\infty$. Since $\left\{m_{\alpha}\right\}$ is monotone nonincreasing, the sequence $\left\{m_{\alpha}\right\}$ converges to 0 .

Let $\varphi: D_{0} \rightarrow N$ be the map given by $\varphi(x)=\bar{x}$ for any $x \in D_{0}$. Then, we have $d(\varphi(x), \varphi(y)) \leq d(x, y)$ for all $x, y \in D_{0}$. Since $D_{0}$ is compact, the metric space $(N, d)$ is compact, and, in particular, complete and locally compact.

For each $\alpha \in \Lambda_{0}$ we define the map $\varphi_{\alpha N}: D_{\alpha} \rightarrow N$ by $\varphi_{\alpha N}\left(x_{\alpha}\right)=\bar{x}$ for any $x_{\alpha} \in D_{\alpha}$ where $x$ is a point in $D_{0}$ with $\varphi_{\alpha}(x)=x_{\alpha}$. Obviously, we have $\varphi_{\alpha N}\left(\varphi_{\alpha}(x)\right)=\varphi(x)=\bar{x}$ for all $x \in D_{0}$ and $d\left(\varphi_{\alpha N}\left(x_{\alpha}\right), \varphi_{\alpha N}\left(y_{\alpha}\right)\right) \leq d\left(x_{\alpha}, y_{\alpha}\right)$ for all $x_{\alpha}, y_{\alpha} \in D_{\alpha}$.

Using Lemma 13 we prove the following.

LemmA 14. Let $x, y \in D_{0}$ and $x_{\alpha}=\varphi_{\alpha}(x) \in D_{\alpha}, y_{\alpha}=\varphi_{\alpha}(y) \in D_{\alpha}$. Let $T_{\alpha}=$ $T\left(x_{\alpha}, y_{\alpha}\right)$ be a minimal geodesic segment connecting $x_{\alpha}$ and $y_{\alpha}$ in $D_{\alpha}$. Then, there exists a subsequence of the sequence $\left\{\varphi_{\alpha N}\left(T_{\alpha}\right)\right\}_{\alpha \in \Lambda_{0}}$ converging to a minimal geodesic segment $\bar{T}$ in $N$, namely $d(\bar{x}, \bar{z})+d(\bar{z}, \bar{y})=d(\bar{x}, \bar{y})$ for every $\bar{z} \in \bar{T}$. In particular, $(N, d)$ is a length space.

PROOF. Since $\varphi_{\alpha N}\left(T_{\alpha}\right)$ are curves connecting $\bar{x}$ and $\bar{y}$ with lengths less than the diameter of $D_{0}$, it follows from Ascoli's theorem that there exists a subsequence $\left\{\varphi_{\alpha_{j} N}\left(T_{\alpha_{j}}\right)\right\}$ of the sequence $\left\{\varphi_{\alpha N}\left(T_{\alpha}\right)\right\}_{\alpha \in \Lambda_{0}}$ converging to a curve $\bar{T}$ in $N$. Let $\bar{z} \in \bar{T}$. There exists a sequence of points $z_{\alpha_{j}} \in T_{\alpha_{j}} \subset D_{\alpha_{j}}$ such that $\left\{\varphi_{\alpha_{j} N}\left(z_{\alpha_{j}}\right)\right\}$ converges to $\bar{z}$ in $N$. It follows from the definition of $z_{\alpha_{j}}$ and Lemma 13 that, for any positive $\varepsilon$, there exists a $j_{0}$ such that $d\left(\varphi_{\alpha_{j} N}\left(z_{\alpha_{j}}\right), \bar{z}\right)<\varepsilon / 3$ and $\left|d\left(x_{\alpha_{j}}, y_{\alpha_{j}}\right)-d(\bar{x}, \bar{y})\right|<\varepsilon / 3$ for all $j \geq j_{0}$. Since

$$
\begin{aligned}
d(\bar{x}, \bar{z}) & \leq d\left(\varphi_{\alpha_{j} N}\left(x_{\alpha_{j}}\right), \varphi_{\alpha_{j} N}\left(z_{\alpha_{j}}\right)\right)+d\left(\varphi_{\alpha_{j} N}\left(z_{\alpha_{j}}\right), \bar{z}\right) \\
& <d\left(x_{\alpha_{j}}, z_{\alpha_{j}}\right)+\varepsilon / 3
\end{aligned}
$$


and

$$
\begin{aligned}
d(\bar{y}, \bar{z}) & \leq d\left(\varphi_{\alpha_{j} N}\left(y_{\alpha_{j}}\right), \varphi_{\alpha_{j} N}\left(z_{\alpha_{j}}\right)\right)+d\left(\varphi_{\alpha_{j} N}\left(z_{\alpha_{j}}\right), \bar{z}\right) \\
& <d\left(y_{\alpha_{j}}, z_{\alpha_{j}}\right)+\varepsilon / 3
\end{aligned}
$$

we have

$$
\begin{aligned}
d(\bar{x}, \bar{y}) & \leq d(\bar{x}, \bar{z})+d(\bar{z}, \bar{y}) \\
& \leq d\left(x_{\alpha_{j}}, z_{\alpha_{j}}\right)+d\left(z_{\alpha_{j}}, y_{\alpha_{j}}\right)+2 \varepsilon / 3 \\
& =d\left(x_{\alpha_{j}}, y_{\alpha_{j}}\right)+2 \varepsilon / 3 \\
& <d(\bar{x}, \bar{y})+\varepsilon .
\end{aligned}
$$

Thus, we have $d(\bar{x}, \bar{z})+d(\bar{z}, \bar{y})=d(\bar{x}, \bar{y})$.

Notice that if $\bar{T}$ passes through a spiral vertex of $N$, then $\bar{T}$ may be broken at that point. In order to study the properties of $\bar{T}$ more, we need to know the topological and metric structure of $N$.

LEMMA 15. $\varphi\left(\partial D_{0}\right)$ is a simple closed curve. All edges in $\varphi\left(\partial D_{0}\right)$ are locally convex and the inner angle at any point in $\varphi\left(\partial D_{0}\right) \backslash \varphi\left(v\left(\partial D_{0}\right)\right)$ is $\pi$.

PROOF. We first prove that $\varphi\left(\partial D_{0}\right)$ is simple. Suppose for indirect proof that $\bar{x}=$ $\varphi(x)=\varphi(y)=\bar{y}$ for some $x, y \in \partial D_{0}$ with $x \neq y$. Let $x \in T_{1}$ and $y \in T_{2}$ where $T_{1}$ and $T_{2}$ are edges contained in $\partial D_{0}$. Suppose as the first case that $x$ is an endpoint $T_{1}$, namely a vertex of $\partial D_{0}$, and $y$ is not the endpoint of $T_{2}=T(p, q)$. There exists an $\alpha \in \Lambda_{0}$ such that $d\left(\varphi_{\alpha}(x), \varphi_{\alpha}(y)\right)<\min \{L(T(p, y)), L(T(y, q))\}$. We have $d\left(\varphi_{\alpha}(x), \varphi_{\alpha}(p)\right)<$ $d\left(\varphi_{\alpha}(p), \varphi_{\alpha}(q)\right)$ and $d\left(\varphi_{\alpha}(x), \varphi_{\alpha}(q)\right)<d\left(\varphi_{\alpha}(p), \varphi_{\alpha}(q)\right)$. Consider a minimum spanning tree $S$ on $v\left(\partial D_{\alpha}\right)$ in $D_{\alpha}$ containing $T_{2}$. Such an $S$ exists because of the condition (P5). Remove $T_{2} \backslash\{p, q\}$ from $S$. Then, $S \backslash\left(T_{2} \backslash\{p, q\}\right)$ consists of two connected components $S(p)$ and $S(q)$ where $S(p)$ and $S(q)$ are the connected components containing $p$ and $q$, respectively. Hence, one of $\left\{\varphi_{\alpha}(x), \varphi_{\alpha}(p)\right\}$ and $\left\{\varphi_{\alpha}(x), \varphi_{\alpha}(q)\right\}$ is contained in a connected component, say $S(p)$. Then the spanning tree $S(p) \cup T\left(\varphi_{\alpha}(x), \varphi_{\alpha}(q)\right) \cup S(q)$ on $v\left(\partial D_{\alpha}\right)$ has its length less than $L(S)$, contradicting the condition (P4).

Next we suppose that both $x$ and $y$ are points in $v\left(\partial D_{0}\right)$. Since we have $\inf _{\alpha \in \Lambda_{0}} d\left(\varphi_{\alpha}(x)\right.$, $\left.\varphi_{\alpha}(y)\right)=0$, there exists an $\alpha_{1} \in \Lambda_{0}$ such that a minimal geodesic segment $T\left(\varphi_{\alpha_{1}}(x), \varphi_{\alpha_{1}}(y)\right)$ must be used in all minimum spanning trees on $v\left(\partial D_{\alpha_{1}}\right)$ in $D_{\alpha_{1}}$. This contradicts that $L\left(r\left(s, D_{\alpha}\right)\right)$ is constant for $\alpha \geq \alpha_{1}$ and $s \in \operatorname{mst}\left(D_{\alpha_{1}}\right)$. Then, there exists an $\alpha_{2} \in \Lambda_{0}$ such that $D_{\alpha_{2}} \in \mathcal{M}_{0}$ does not satisfy the conditions (P3) and (P4).

Next we suppose that both $x$ and $y$ are not the endpoints of $T_{1}$ and $T_{2}$, respectively. Then, $x$ and $y$ divide $T_{1}$ and $T_{2}$ into two minimal geodesic segments, respectively. Let $2 \delta$ be the minimum value of all the lengths of these segments. Since $\varphi(x)=\varphi(y)$, there exists an $\alpha_{0} \in \Lambda_{0}$ such that there exists a minimal geodesic segment $T\left(\varphi_{\alpha}(x), \varphi_{\alpha}(y)\right)$ passing through the interior of $D_{\alpha}$ for any $\alpha \geq \alpha_{0}$. Then $D_{\alpha}$ is divided into two connected components by $T\left(\varphi_{\alpha}(x), \varphi_{\alpha}(y)\right)$. We say that $x^{\prime} \in T_{1}$ and $y^{\prime} \in T_{2}$ are in the same side with respect to 
$x, y$ in the edges if any minimal geodesic segment connecting $x^{\prime}$ and $y^{\prime}$ does not intersect $T\left(\varphi_{\alpha}(x), \varphi_{\alpha}(y)\right)$ for every $\alpha \geq \alpha_{0}$. We claim that $\delta$ satisfies that $\varphi\left(x^{\prime}\right)=\varphi\left(y^{\prime}\right)$ for $x^{\prime} \in T_{1}$ and $y^{\prime} \in T_{2}$ if $d\left(x, x^{\prime}\right)=d\left(y, y^{\prime}\right)<\delta$ and $x^{\prime}, y^{\prime}$ are in the same side with respect to $x, y$ in the edges. In fact, if $\bar{x}^{\prime}=\varphi\left(x^{\prime}\right) \neq \varphi\left(y^{\prime}\right)=\bar{y}^{\prime}$ for some $x^{\prime}$ and $y^{\prime}$, we then take a point $x^{\prime \prime}$ with $d\left(x^{\prime \prime}, x\right)<\delta$ in the other side of $x^{\prime}$ with respect to $x$ in $T_{1}$, namely, $x^{\prime \prime}$, $x$ and $x^{\prime}$ are in this order on $T_{1}$, and consider the comparison triangle domain $\widetilde{\triangle}\left(\bar{x}^{\prime \prime} \bar{x}^{\prime} \bar{y}^{\prime}\right)$ in $M(\kappa)$ corresponding to $\triangle\left(\bar{x}^{\prime \prime} \bar{x}^{\prime} \bar{y}^{\prime}\right)$. Since $d\left(\bar{x}^{\prime}, \bar{y}^{\prime}\right)>0, \widetilde{\triangle}\left(\bar{x}^{\prime \prime} \bar{x}^{\prime} \bar{y}^{\prime}\right)$ is not degenerate. So are $\widetilde{\triangle}\left(\varphi_{\alpha}\left(x^{\prime \prime}\right) \varphi_{\alpha}\left(x^{\prime}\right) \varphi_{\alpha}\left(y^{\prime}\right)\right)$ and $\triangle\left(\varphi_{\alpha}\left(x^{\prime \prime}\right) \varphi_{\alpha}\left(x^{\prime}\right) \varphi_{\alpha}\left(y^{\prime}\right)\right)$ because of Lemma 13, and, in particular, $\triangle\left(\bar{x}^{\prime \prime} \bar{x}^{\prime} \bar{y}^{\prime}\right)$ is not degenerate because of Lemma 13. This implies that $d(\bar{x}, \bar{y})>0$, a contradiction. Here we give the detail of the estimates of the distances. For any $\alpha_{0} \in \Lambda_{0}$, there exists an $\alpha \in \Lambda_{0}$ with $\alpha \geq \alpha_{0}$ such that if $x_{\alpha}^{\prime} \in T\left(\widetilde{\varphi_{\alpha}\left(x^{\prime \prime}\right)}, \widetilde{\varphi_{\alpha}\left(x^{\prime}\right)}\right)$ is the point corresponding to $x$ with $d\left(x^{\prime \prime}, x\right)=d\left(\widehat{\varphi_{\alpha}\left(x^{\prime \prime}\right)}, x_{\alpha}^{\prime}\right)$ in $\widetilde{\triangle}\left(\varphi_{\alpha}\left(x^{\prime \prime}\right) \varphi_{\alpha}\left(x^{\prime}\right) \varphi_{\alpha}\left(y^{\prime}\right)\right)$, then

$$
d\left(\varphi_{\alpha}(x), \varphi_{\alpha}(y)\right) \geq d\left(\varphi_{\alpha}(x), T\left(\varphi_{\alpha}\left(x^{\prime \prime}\right), \varphi_{\alpha}\left(y^{\prime}\right)\right)\right) \geq d\left(x_{\alpha}^{\prime}, T\left(\widetilde{\varphi_{\alpha}\left(x^{\prime \prime}\right)}, \widetilde{\varphi_{\alpha}\left(y^{\prime}\right)}\right)\right) .
$$

In fact, since $\varphi_{\alpha}\left(T_{1}\right)$ and $\varphi_{\alpha}\left(T_{2}\right)$ are minimal geodesic segments in $D_{\alpha}$ and locally convex, there exists an $\alpha \in \Lambda_{0}$ with $\alpha \geq \alpha_{0}$ such that the minimal geodesic segment $T\left(\varphi_{\alpha}\left(x^{\prime \prime}\right)\right.$, $\left.\varphi_{\alpha}\left(y^{\prime}\right)\right) \backslash\left\{\varphi_{\alpha}\left(x^{\prime \prime}\right), \varphi_{\alpha}\left(y^{\prime}\right)\right\}$ is contained in Int $D_{\alpha}$ and $T\left(\varphi_{\alpha}\left(x^{\prime \prime}\right), \varphi_{\alpha}\left(y^{\prime}\right)\right)$ divides $D_{\alpha}$ into two connected components, say $\varphi_{\alpha}(x) \in D_{\alpha 1}$ and $\varphi_{\alpha}(y) \in D_{\alpha 2}$. Since $\triangle\left(\varphi_{\alpha}\left(x^{\prime \prime}\right) \varphi_{\alpha}\left(x^{\prime}\right) \varphi_{\alpha}\left(y^{\prime}\right)\right) \subset$ $D_{\alpha 1}, \varphi_{\alpha}(y) \in D_{\alpha 2}$ and $D_{\alpha 1} \cap D_{\alpha 2}=T\left(\varphi_{\alpha}\left(y^{\prime}\right), \varphi_{\alpha}\left(x^{\prime \prime}\right)\right)$, we have the first inequality. Lemma $3\left(\mathrm{~A}^{\prime}\right)$ shows the second inequality. Thus we have, from Lemma 13,

$$
d(\bar{x}, \bar{y}) \geq \inf _{\alpha \in \Lambda_{0}} d\left(x_{\alpha}^{\prime}, T\left(\widetilde{\varphi_{\alpha}\left(x^{\prime \prime}\right)}, \widetilde{\varphi_{\alpha}\left(y^{\prime}\right)}\right)\right)=d\left(x_{0}^{\prime}, T\left(\widetilde{\bar{x}^{\prime \prime}}, \widetilde{\bar{y}^{\prime}}\right)\right)>0,
$$

where $x_{0}^{\prime}$ is the point corresponding to $x$ with $d\left(x^{\prime \prime}, x\right)=d\left(\bar{x}^{\prime \prime}, x_{0}^{\prime}\right)$ in $\widetilde{\triangle}\left(\bar{x}^{\prime \prime} \bar{x}^{\prime} \bar{y}^{\prime}\right)$. This inequality contradicts that $\inf _{\alpha \in \Lambda_{0}} d\left(\varphi_{\alpha}(x), \varphi_{\alpha}(y)\right)=d(\bar{x}, \bar{y})=0$. Therefore, it follows from the claim that an endpoint of one of $T_{1}$ and $T_{2}$ converges to an interior point or an endpoint of the other. Hence, this case is included in the previous cases. As a result we have a contradiction if we suppose $\varphi: \partial D_{0} \rightarrow N$ is not injective.

We prove the second part of the lemma. It follows from Lemma 14 that all edges in $\varphi\left(\partial D_{0}\right)$ are minimal. Suppose that an edge $T_{2}$ in $\varphi\left(\partial D_{0}\right)$ is not locally convex. Then there exists a minimal geodesic segment $T_{1}$ in $N$ such that $\bar{x}=\bar{y}$ for some $\bar{x} \in \operatorname{Int} T_{1}$ and $\bar{y} \in \operatorname{Int} T_{2}$ and $T_{1} \neq T_{2}$ in any neighborhood of $\bar{x}$. On the other hand, as was seen in the proof of the first part, $T_{1}$ and $T_{2}$ coincide in some neighborhood of the intersection, a contradiction.

Since $\varphi\left(\partial D_{0}\right)$ are locally convex, the inner angle at any interior point in any edge of $\varphi\left(\partial D_{0}\right)$ is $\pi$.

We call $\varphi\left(\partial D_{0}\right)$ the boundary of $N$ and it is denoted by $\partial N$. We prove that $N \backslash \varphi\left(\partial D_{0}\right) \neq$ $\emptyset$.

LEMMA 16. $N \backslash \partial N$ is not empty. Each edge in $\partial N$ is the limit of a sequence of minimal geodesic segments in $N \backslash \partial N$. 
PRoOF. Let $g_{\alpha}=\max \left\{d\left(x, \partial D_{\alpha}\right) ; x \in D_{\alpha}\right\}$ for every $\alpha \in \Lambda_{0}$. We have only to prove that there exists a positive $\delta$ such that $g_{\alpha} \geq \delta$ for all $\alpha \in \Lambda_{0}$. Suppose for indirect proof that $\inf _{\alpha \in \Lambda_{0}} g_{\alpha}=0$. Then, $N=\partial N$. Let a point $p \in \partial D_{0}$ be fixed and $h:[0,1] \times[0,1] \rightarrow D_{0}$ be a continuous map such that $h(0, \cdot):[0,1] \rightarrow \partial D_{0}$ is a parametrization of $\partial D_{0}$ with $h(0,0)=h(0,1)=p$ and $h(1,[0,1])=p$. Then, $\varphi \circ h:[0,1] \times[0,1] \rightarrow N$ gives a homotopy from $\partial N$ to the point $\varphi(p)$ in $N$. This implies that $\partial N$ is simply connected, contradicting Lemma 15.

Since all edges in $\partial N$ are locally convex and minimal, the second part of the lemma holds.

We call $N \backslash \partial N$ the interior of $N$ and it is denoted by Int $N$. The next step is to prove that Int $N$ is an Alexandrov surface with curvature bounded below by $\kappa$.

Let $B(x, r)$ be the open metric ball with center $x$ and radius $r$, namely, $B(x, r)$ is the set of all points $y$ with $d(x, y)<r$.

LeMma 17. Let $\bar{x}$ be a point in $N$ with $B(\bar{x}, r) \subset \operatorname{Int} N$. Let $\varphi(x)=\bar{x}$. Then, $B\left(\varphi_{\alpha}(x), r\right) \subset$ Int $D_{\alpha}$ for any $\alpha \in \Lambda_{0}$.

PROOF. Suppose $B\left(\varphi_{\alpha}(x), r\right) \cap \partial D_{\alpha} \neq \emptyset$ for some $\alpha$, say $\varphi_{\alpha}\left(y_{\alpha}\right) \in B\left(\varphi_{\alpha}(x), r\right) \cap \partial D_{\alpha}$, $y_{\alpha} \in D_{0}$. Since $\mathcal{M}_{0}$ is a totally ordered set, for $\beta \geq \alpha$ we have $\varphi_{\beta}\left(y_{\alpha}\right)=\varphi_{\alpha \beta}\left(\varphi_{\alpha}\left(y_{\alpha}\right)\right) \in$ $\partial D_{\beta}$, and hence $\bar{y}_{\alpha} \in \partial N$. On the other hand, $d\left(\bar{x}, \bar{y}_{\alpha}\right)=\inf _{\beta \geq \alpha} d\left(\varphi_{\beta}(x), \varphi_{\beta}\left(y_{\alpha}\right)\right) \leq$ $d\left(x, y_{\alpha}\right)<r$, namely, $\bar{y}_{\alpha} \in B(\bar{x}, r)$, contradicting the assumption $B(\bar{x}, r) \subset \operatorname{Int} N$.

Lemma 18. Let $\bar{x} \in \operatorname{Int} N$ with $B(\bar{x}, 5 r) \subset \operatorname{Int} N$ and let $x \in D_{0}$ with $\varphi(x)=\bar{x}$. Let $\bar{p}, \bar{q}$ be points such that $\bar{p}, \bar{q} \in B(\bar{x}, r)$. Then, there exists an $\alpha_{0} \in \Lambda_{0}$ such that a minimal geodesic segment $T\left(\varphi_{\alpha}(p), \varphi_{\alpha}(q)\right)$ is contained in $B\left(\varphi_{\alpha}(x), 4 r\right) \subset D_{\alpha}$ for any $\alpha>\alpha_{0}$.

Proof. From Lemma 13, there exists an $\alpha_{0} \in \Lambda_{0}$ such that $d\left(\varphi_{\alpha}(y), \varphi_{\alpha}(z)\right)-d(\bar{y}, \bar{z})<$ $r$ for any $\alpha \geq \alpha_{0}$, and for any $y, z \in D_{0}$. For those $\alpha$ we have

$$
d\left(\varphi_{\alpha}(x), \varphi_{\alpha}(p)\right) \leq d(\bar{x}, \bar{p})+r<2 r
$$

and

$$
\begin{aligned}
d\left(\varphi_{\alpha}(p), \varphi_{\alpha}(q)\right) & \leq d(\bar{p}, \bar{q})+r \\
& \leq d(\bar{p}, \bar{x})+d(\bar{x}, \bar{q})+r \leq 3 r .
\end{aligned}
$$

Hence, for any $\varphi_{\alpha}(w) \in T\left(\varphi_{\alpha}(p), \varphi_{\alpha}(q)\right)$ with $w \in D_{0}$,

$$
\begin{aligned}
& d\left(\varphi_{\alpha}(x), \varphi_{\alpha}(w)\right) \\
& \quad \leq \frac{1}{2}\left\{d\left(\varphi_{\alpha}(x), \varphi_{\alpha}(p)\right)+d\left(\varphi_{\alpha}(p), \varphi_{\alpha}(q)\right)+d\left(\varphi_{\alpha}(q), \varphi_{\alpha}(x)\right)\right\} \\
& \quad<4 r .
\end{aligned}
$$

LEMma 19. (Int $N, d)$ is an Alexandrov space with curvature bounded below by $\kappa$. 
PROOF. Let $\bar{x}$ and $r$ be as in Lemma 18. Let $\triangle(\bar{p} \bar{q} \bar{r})$ be a geodesic triangle domain in $B(\bar{x}, r)$ and $\triangle(\tilde{p} \tilde{q} \tilde{r})$ a comparison triangle domain in $M(\kappa)$ corresponding to $\triangle(\bar{p} \bar{q} \bar{r})$. Let $\bar{s} \in T(\bar{q}, \bar{r})$ and let $s^{\prime} \in T(\tilde{q}, \tilde{r})$ be the point corresponding to $\bar{s}$. Since Int $D_{\alpha}$ is an Alexandrov surface, the condition $(\mathrm{C})$ shows that

$$
\angle \widetilde{\left(\varphi_{\alpha}(q)\right.} \widetilde{\varphi_{\alpha}(s)} \widetilde{\left.\varphi_{\alpha}(r)\right)} \leq L\left(\varphi_{\alpha}(q) \varphi_{\alpha}(s) \varphi_{\alpha}(r)\right) \leq \pi .
$$

Since

$$
d(\bar{q}, \bar{r})=d(\bar{q}, \bar{s})+d(\bar{s}, \bar{r})=\lim _{\alpha \in \Lambda_{0}}\left\{d\left(\varphi_{\alpha}(q), \varphi_{\alpha}(s)\right)+d\left(\varphi_{\alpha}(s), \varphi_{\alpha}(r)\right)\right\},
$$

the sequence of angles $\angle \widetilde{\left(\varphi_{\alpha}(q)\right.} \widetilde{\varphi_{\alpha}(s)} \widetilde{\left.\varphi_{\alpha}(r)\right)}$ converges to $\pi$. Therefore, the sequence of angles $\angle\left(\varphi_{\alpha}(q) \varphi_{\alpha}(s) \varphi_{\alpha}(r)\right)$ converges to $\pi$ also. Since Int $D_{\alpha}$ is an Alexandrov surface and by Lemma 3 (D), we have

$$
2 \pi \geq L\left(\widetilde{\varphi_{\alpha}(p)} \widetilde{\varphi_{\alpha}(s)} \widetilde{\left.\varphi_{\alpha}(r)\right)}+L\left(\widetilde{\left(\varphi_{\alpha}(p)\right.} \widetilde{\varphi_{\alpha}(s)} \widetilde{\left.\varphi_{\alpha}(q)\right)}+L \widetilde{\left(\varphi_{\alpha}(q) \widetilde{\varphi_{\alpha}(s)}\right.} \widetilde{\left.\varphi_{\alpha}(r)\right)} .\right.\right.
$$

Therefore, we have

$$
\limsup _{\alpha \in \Lambda_{0}}\left\{\left(\widetilde{\varphi_{\alpha}(p)} \widetilde{\varphi_{\alpha}(s)} \widetilde{\left.\varphi_{\alpha}(r)\right)}+\angle \widetilde{\left.\left(\widetilde{\varphi_{\alpha}(p)} \widetilde{\varphi_{\alpha}(s)} \widetilde{\varphi_{\alpha}(q)}\right)\right\}} \leq \pi .\right.\right.
$$

Hence, if a quadrangle $\tilde{\bar{p}} \tilde{\bar{q}} \tilde{\bar{s}} \tilde{\bar{s}}$ is the union of comparison triangle domains $\widetilde{\triangle}(\bar{p} \bar{q} \bar{s})$ and $\widetilde{\triangle}(\bar{p} \bar{r} \bar{s})$ glued along $T(\tilde{\bar{p}}, \tilde{\bar{s}})$, then the angle of the quadrangle $\tilde{\bar{p}} \tilde{\bar{q}} \tilde{\bar{s}} \tilde{\bar{r}}$ at $\tilde{\bar{s}}$ is less than or equal to $\pi$. Therefore, $d(\bar{p}, \bar{s}) \geq d\left(\tilde{p}, s^{\prime}\right)$.

We need to prove that $N$ is a surface homeomorphic to a disk.

LEMMA 20. $N$ is homeomorphic to a disk.

PROOF. We have only to prove that there exists an $\alpha_{0} \in \Lambda_{0}$ such that $N$ is homeomorphic to $D_{\alpha}$ for all $\alpha>\alpha_{0}$, because $D_{\alpha}$ is homeomorphic to a disk. Since $\varphi$ does not increase the distance, we have $\operatorname{dim} N \leq 2$. Since $\operatorname{Int} N \neq \emptyset$, we have $\operatorname{dim} N \neq 1$. Hence, $\operatorname{dim} N=2$. This implies that $N$ is a topological surface with boundary $\partial N$. Take a positive $\theta$ such that all geodesic triangle domains with diameter less than $\theta$ in $N$ are homeomorphic to disks. Let $\Sigma$ be a triangulation of $N$ such that the diameters of all geodesic triangle domains in $\Sigma$ is less than $\theta$. Let $\bar{x}_{i}, i=1, \ldots, k$, be the vertices of the triangulation $\Sigma$ and $x_{i} \in D_{0}$ the points with $\varphi\left(x_{i}\right)=\bar{x}_{i}$. It follows from Lemma 14 that for any positive $\varepsilon$ there exists an $\alpha_{1} \in \Lambda_{0}$ such that $\varphi_{\alpha N}\left(T_{\alpha}\right)$ is contained in the $\varepsilon$-neighborhood of $\bar{T}=T(\bar{x}, \bar{y})$ if $\bar{T}$ is some minimal geodesic segment connecting $\bar{x}$ and $\bar{y}$ in $N$ and $T_{\alpha}$ is a minimal geodesic segment connecting $x_{\alpha}=\varphi_{\alpha}(x)$ and $y_{\alpha}=\varphi_{\alpha}(y)$ in $D_{\alpha}$ if $\alpha \geq \alpha_{1}$. Therefore, we have the triangulation $\Sigma_{\alpha}$ of $D_{\alpha}$ whose vertices are $\left(x_{i}\right)_{\alpha}, i=1, \ldots, k$. Thus there exists an $\alpha_{0} \in \Lambda_{0}$ such that the triangulation $\Sigma_{\alpha}$ is homeomorphic to $\Sigma$ as a graph if $\alpha$ satisfies $\alpha \geq \alpha_{0} \geq \alpha_{1}$. Since $D_{\alpha}$ is topologically a disk, each triangle in $\Sigma_{\alpha}$ surrounds a domain which is homeomorphic to a disk. From this we see that $N$ is homeomorphic to a disk $D_{\alpha}$ if $\alpha \in \Lambda_{0}$ satisfies $\alpha>\alpha_{0}$.

Thus, $N$ is a polygonal surface with curvature bounded below by $\kappa$. We next prove that $N$ is an upper bound of $\mathcal{M}_{0}$. 
Lemma 21. $(N, d)$ is contained in $\mathcal{M}$ and $D_{\alpha} \leq N$ for all $\alpha \in \Lambda_{0}$.

PROOF. The condition (P1) is satisfied by $N$ because of the construction and Lemma 14 . Let $\varphi: D_{0} \rightarrow N$ be given by $\varphi(x)=\bar{x}$. Obviously, it follows that $d(\varphi(x), \varphi(y)) \leq d(x, y)$ for any $x, y \in D_{0}$. Let $x$ and $y$ be points in an edge of the boundary $\partial D_{0}$ of $D_{0}$. Then,

$$
d(\varphi(x), \varphi(y))=d(\bar{x}, \bar{y})=\inf _{\alpha \in \Lambda_{0}} d\left(\varphi_{\alpha}(x), \varphi_{\alpha}(y)\right)=d(x, y) .
$$

This implies that $N$ satisfies the condition (P2). We prove the condition (P4). Let $s \in$ $\operatorname{mst}\left(D_{0}\right) \subset \operatorname{mst}\left(D_{\alpha}\right)$ and let $T_{1}, \ldots, T_{n-1}$ be minimal geodesic segments which are edges of $r\left(s, D_{0}\right)$. Since $L\left(\varphi_{\alpha}\left(T_{k}\right)\right) \leq L\left(T_{k}\right)$ and

$$
L\left(r\left(s, D_{\alpha}\right)\right) \leq \sum_{k=1}^{n-1} L\left(\varphi_{\alpha}\left(T_{k}\right)\right) \leq \sum_{k=1}^{n-1} L\left(T_{k}\right)=L\left(r\left(s, D_{0}\right)\right),
$$

we have $L\left(\varphi_{\alpha}\left(T_{k}\right)\right)=L\left(T_{k}\right)$ for all $k=1, \ldots, n-1$, and $\varphi_{\alpha}\left(T_{k}\right)$ is a minimal geodesic segment for $k=1, \ldots, n-1$. Therefore, $\varphi\left(T_{k}\right)$ is also a minimal geodesic segment for all $k=1, \ldots, n-1$ and $L(r(s, N))=L\left(r\left(s, D_{0}\right)\right)$. The condition (P4) implies (P5) for $N$. In order to prove the condition $(\mathrm{P} 3)$, we suppose that $\operatorname{mst}(N)$ does not contain $\operatorname{mst}\left(D_{0}\right)$. Then, there exists a minimum spanning tree $T$ on $v(\partial N)$ in $N$ such that $L(T)<L(r(s, N)) \leq$ $L\left(r\left(s, D_{0}\right)\right)$ for any $s \in \operatorname{mst}\left(D_{0}\right)$. Let $t$ be a topology of $T$. Then, since

$$
L(T)=\inf _{\alpha \in \Lambda_{0}} L\left(r\left(t, D_{\alpha}\right)\right),
$$

there exists an $\alpha_{0} \in \Lambda_{0}$ with $L\left(r\left(t, D_{\alpha}\right)\right)<L\left(r\left(s, D_{\alpha}\right)\right)$ for any $\alpha \geq \alpha_{0}$, contradicting the condition $\operatorname{mst}\left(D_{\alpha}\right) \supset \operatorname{mst}\left(D_{0}\right)$ for $\alpha \in \Lambda_{0}$. At the end of the proof, we notice that $\varphi_{\alpha N}: D_{\alpha} \rightarrow N$ for any $\alpha \in \Lambda_{0}$ is a compression map.

We have proved the following because of Zorn's lemma.

LEMma 22. $(\mathcal{M}, \leq)$ is an inductively partially ordered set. Therefore, there exists a maximum element in $\mathcal{M}$.

5. Proof of Theorem 1. In this section we prove Theorem 1. Let $D$ be the polygonal surface as in Theorem 1 . We change $D$ into a polygonal surface $D_{0}$ as in Lemma 10 . As was seen in Lemma 22 , we have the inductively ordered set $(\mathcal{M}, \leq)$ from the polygonal surface $D_{0}$. Let $\widetilde{D}$ be a maximum element in $\mathcal{M}$. The polygonal surfaces $D$ and $\widetilde{D}$ satisfy the properties about SMT and MST stated in Theorem 1 because of the construction. We have to prove that Int $\widetilde{D}$ is smooth and has constant curvature $\kappa$. This follows from Lemma 23.

LEMMA 23. If $\widetilde{D} \in \mathcal{M}$ is not a smooth polygonal surface with constant curvature $\kappa$, then there exists an element $D_{1} \in \mathcal{M}$ with $\widetilde{D}<D_{1}$.

Proof. Let $p$ be a point in Int $\widetilde{D}$ such that any neighborhood around $p$ is not isometric to a subset in $M(\kappa)$. We have two cases, namely, $p \in \Gamma(\widetilde{D})$ or $p \in \widetilde{D} \backslash \Gamma(\widetilde{D})$, where $\Gamma(\widetilde{D})$ is the union of all minimum spanning trees $r(s, \widetilde{D}), s \in \operatorname{mst}(\widetilde{D})$. If $p \in \widetilde{D} \backslash \Gamma(\widetilde{D})$, then there exists a sufficiently small geodesic triangle domain $\triangle$ around $p$ which is contained in 
$\widetilde{D} \backslash \Gamma(\widetilde{D})$ such that any minimum spanning tree on $v(\partial \widetilde{D})$ in $D_{1}=(\widetilde{D} \backslash \triangle) \cup \tilde{\triangle}$ does not intersect $\triangle$, where $D_{1}$ is a polygonal surface given as follows. $\triangle$ is removed from $\widetilde{D}$ and a comparison triangle domain $\widetilde{\triangle}$ in $M(\kappa)$ is glued on $\widetilde{D} \backslash \triangle$ along its edges. Then, $D_{1} \in \mathcal{M}$ with $\widetilde{D}<D_{1}$ because of Lemma 5. Assume that $p \in \Gamma(\widetilde{D})$. Let $S$ be the edge of $\Gamma(\widetilde{D})$ containing $p$. We should notice that $p$ is not a singular point because Int $\widetilde{D}$ is an Alexandrov surface with curvature bounded below by $\kappa$, and hence there exists a point $q$ near $p$ such that any neighborhood around $q$ is not isometric to a subset in $M(\kappa)$. If we can find a point $q \notin S$ near $p$ such that some neighborhood around $q$ is not isometric to any subset in $M(\kappa)$ and does not intersect any biangle domain one of whose sides is $S$. By using the point $q$ instead of $p$ as in the above argument, we can see that $D_{1} \in \mathcal{M}$ exists with $\widetilde{D}<D_{1}$. Otherwise, there exists a thin biangle domain $V$ one of whose sides is $S$, then Lemma 6 shows that $D_{1} \in \mathcal{M}$ with $\widetilde{D}<D_{1}$ exists.

The review of our method is helpful to understand the process of deformation, in particular, what happens on the condition (P3). Roughly speaking, Lemmas 5 and 6, combined with Lemma 9 (3), states that we can deform a polygonal surface $D_{0}$ with $\partial D_{0} \subset \Gamma\left(D_{0}\right)$ continuously and separately in each connected component of $D_{0} \backslash \Gamma\left(D_{0}\right)$, leaving the set $\operatorname{mst}\left(D_{0}\right)$ of topologies of minimum spanning trees on $v\left(\partial D_{0}\right)$ invariant. We continue the deformation until we cannot do it anymore in this way. The changing polygonal surface yields a new edge which does not degenerate. If $D^{\prime}$ is the resulting polygonal surface changed from $D_{0}$, then $D^{\prime}$ must be a smooth polygonal surface with constant curvature $\kappa$ or $\operatorname{mst}\left(D^{\prime}\right) \supsetneq \operatorname{mst}\left(D_{0}\right)$, meaning that the new edge used in some minimum spanning tree appears. Due to the continuous property of deformation and Lemma 9 (3), the new edge dose not cross the old ones. Zorn's lemma states that this time comes, the existence of a maximum element. In the first case, the deformation completes. In the second case, the deformation starts again from $D^{\prime}$, leaving $\operatorname{mst}\left(D^{\prime}\right)$ invariant. This operation will stop after a finite number of iterations, since the number of the topologies of minimum spanning trees on $v\left(\partial D_{0}\right)$ is finite.

6. Characteristic domain. In this section we show how to construct a polygonal surface containing given Steiner minimum tree in a strongly convex domain of a complete Alexandrov surface with curvature bounded below by $\kappa$. We will call such a polygonal surface the characteristic domain.

We describe the characteristic domain a little more before we will show how to construct it. Let $M$ be a complete Alexandrov surface with curvature bounded below by $\kappa$ having no boundary and $M_{0}$ a strongly convex set in $M$. Then, $M_{0}$ is simply connected and smooth. Let $P$ be a set of $n$ points in $M_{0}$. Assume that a Steiner minimum tree $T_{0}=\operatorname{SMT}_{M}(P)$ is full. When $T_{0}$ is not full, we define the characteristic domain of $T_{0}$ by the union of all the characteristic domains of all full Steiner subtrees of $T_{0}$. The characteristic domain $C\left(T_{0}\right)$ of $T_{0}$ is a polygonal surface with curvature bounded below by $\kappa$ such that it includes Int $T_{0}$ in its interior and its vertices are points of $P$, namely $v\left(C\left(T_{0}\right)\right)=P$. A diagonal of a polygonal surface $D$ is by definition a minimal (but not broken) geodesic segment connecting two vertices in the polygonal surface $D$ which is not contained in $\partial D$. Although we do not know whether 
there exists a diagonal of $D$, in general, an important property is that $D$ is divided into two polygonal surfaces by any diagonal of $D$.

Here is how to construct the characteristic domain $C\left(T_{0}\right)$. The construction is the same as Ivanov and Tuzhilin's in [12], since the minimal geodesic segments connecting their endpoints are unique in the strongly convex domain $M_{0}$. Let $p$ and $q$ in $P$ be adjacent points which are by definition connected by a convex subtree $S(p, q)$ of $T_{0}$. Let $T(p, q)$ be the unique minimal geodesic segment connecting $p$ and $q$. If $S(p, q) \cup T(p, q)$ is a simple polygon, then the domain surrounded by $S(p, q) \cup T(p, q)$ is denoted by $D(p, q)$. If $S(p, q) \cup T(p, q)$ is not a simple polygon, then we construct a simply connected spiral domain $D(p, q)$ in the following way. Let $p=p_{0}, p_{1}, \ldots, p_{k}=q$ be self-intersection points of $T(p, q) \cup S(p, q)$ which lie in this order on $T(p, q)$. Since $M_{0}$ is strongly convex and $S(p, q)$ is a convex subtree, at least one of the following is true.

(1) Every subtree $S\left(p_{i}, p_{k}\right)$ of $S(p, q)$ is contained in the simply connected domain surrounded by $T\left(p_{i-1}, p_{i}\right) \cup S\left(p_{i-1}, p_{i}\right)$ for $i=1, \ldots, k-1$.

(2) Every subtree $S\left(p_{i}, p_{k}\right)$ of $S(p, q)$ is in the outside of the simply connected domain surrounded by $T\left(p_{i-1}, p_{i}\right) \cup S\left(p_{i-1}, p_{i}\right)$ for $i=1, \ldots, k-1$.

We say that $q$ (resp. $p$ ) is the center of the spiral $S(p, q)$ if (1) (resp. (2)) happens. At least one of $p$ and $q$ is the center of the spiral $S(p, q)$. There exists an integer $l$ with $0 \leq l<k-1$ such that at least one of $S\left(p_{0}, p_{l}\right)$ and $S\left(p_{l}, p_{k}\right)$ is a spiral around $p$ or $q$, respectively. We assume that $S\left(p_{l}, p_{k}\right)$ is a spiral around $q$. Let $A_{i}$ be the polygonal domain surrounded by $S\left(p_{i}, p_{i+1}\right) \cup T\left(p_{i}, p_{i+1}\right)$ for each $i=l, \ldots, k-1$. Cut $A_{i}$ along the segment $T\left(p_{i+1}, q\right)$, and we have a polygonal domain $A_{i}^{\prime}$ surrounded by the polygon $T\left(q, p_{i}\right) \cup S\left(p_{i}, p_{i+1}^{\prime}\right) \cup$ $T\left(p_{i+1}^{\prime}, q\right)$, where $T\left(p_{i+1}^{\prime}, q\right)$ is a copy of $T\left(p_{i+1}, q\right)$ along which the polygonal domain is cut. Glue $A_{i}^{\prime}$ on $A_{i+1}^{\prime}$ along the segments $T\left(p_{i+1}^{\prime}, q\right)$ and $T\left(p_{i+1}, q\right)$ for all $i=l, \ldots, k-1$. If $l>0$, then we do the same thing in order to construct a spiral domain around $p$. Thus we get the domain $D(p, q)$ which is spiral around at most two points and is simply connected. Finally we glue these domains along the Steiner minimum tree $T_{0}$, and the resulting domain $C\left(T_{0}\right)$ is by definition the characteristic domain of $T_{0}$. The characteristic domain $C\left(T_{0}\right)$ is uniquely determined by $T_{0}$ but not $P$.

We call $D(p, q)$ the basic domain of an adjacent pair of points $p$ and $q$ in $T_{0}$. Let $D(p, q)$ be a basic domain, where $p$ and $q$ are adjacent points in $T_{0}$. Then, $D(p, q)$ is a polygonal surface with curvature bounded below by $\kappa$. Let $p=p_{0}, \ldots, p_{k}=q$ be vertices of the boundary $\partial D(p, q)$ which lie in this order. Notice that the inner angle at $p_{i}$ is $2 \pi / 3$ for every $i=1, \ldots, k-1$. Hence, there exists a integer $k^{\prime}$ with $1 \leq k^{\prime} \leq k-1$ such that the minimal geodesic segment from $p_{0}$ to $p_{i}$ in $D(p, q)$ does not pass through $q$ for every $i=1, \ldots, k^{\prime}$ and it is broken at the point $q$ for every $i=k^{\prime}+1, \ldots, k$. We then have a triangulation of $D(p, q)$ which consists of geodesic triangle domains $\triangle\left(p_{0} p_{i} p_{i+1}\right)$ for $i=1, \ldots, k^{\prime}-1, \triangle\left(p p_{k^{\prime}} q\right), \triangle\left(q p_{k^{\prime}} p_{k^{\prime}+1}\right)$, and $\triangle\left(q p_{i} p_{i+1}\right)$ for $i=k^{\prime}+1, \ldots, k-1$. Combining these triangulations of basic domains we get a triangulation of the characteristic domain of $T_{0}$.

We summarize some properties of the characteristic domain $C\left(T_{0}\right)$. 
PROPOSITION 24. Let $M_{0}$ be a strongly convex set in a complete Alexandrov surface $M$ with curvature bounded below by $\kappa$ having no boundary and $P$ a finite set of points in $M_{0}$. Assume that $T_{0}=\mathrm{SMT}_{M}(P)$ is full. Then, the characteristic domain $D=C\left(T_{0}\right)$ satisfies the following.

(1) The boundary $\partial D$ of $D$ is a polygon whose sides are minimal geodesic segments connecting adjacent points in $P$ which is the set of all vertices of $\partial D$. In particular, each side in $\partial D$ is locally convex.

(2) The interior Int $D$ of $D$ is an Alexandrov surface with curvature bounded below by $\kappa$.

(3) A shortest network interconnecting $v(\partial D)$ contained in $D$ is a Steiner minimum tree for $P$ in $M_{0}$.

(4) The characteristic domain D has a triangulation such that each geodesic triangle domain is contained in a basic domain.

It follows from (1) and (2) that the characteristic domain $C\left(T_{0}\right)$ is a polygonal surface with curvature bounded below by $\kappa$ which is immersed in $M$. The property (3) implies $L\left(T_{0}\right)=L(\operatorname{SMT}(D))$, and hence $\rho\left(M_{0}\right) \geq \eta\left(M_{0}\right)$, which is needed in the proof of Theorem 2. In fact, this inequality is proved as follows. Let $T$ be an Steiner minimum tree for a finite set $P$ of points in $M_{0}$. We decompose $T$ into some full Steiner trees $T_{1}, \ldots, T_{n}$ which are subtrees of $T$. Since the characteristic domains $C\left(T_{k}\right)$ are polygonal surfaces, it holds that $L\left(\operatorname{SMT}\left(C\left(T_{k}\right)\right)\right) \geq \eta\left(M_{0}\right) L\left(\operatorname{MST}\left(C\left(T_{k}\right)\right)\right)$. We then have

and

$$
L(T)=\sum_{k=1}^{n} L\left(T_{k}\right)=\sum_{k=1}^{n} L\left(\operatorname{SMT}\left(C\left(T_{k}\right)\right)\right) \geq \eta\left(M_{0}\right) \sum_{k=1}^{n} L\left(\operatorname{MST}\left(C\left(T_{k}\right)\right)\right),
$$

$$
L\left(\operatorname{MST}_{M_{0}}(P)\right) \leq \sum_{k=1}^{n} L\left(\operatorname{MST}\left(C\left(T_{k}\right)\right)\right),
$$

because $\bigcup_{k=1}^{n} \operatorname{MST}\left(C\left(T_{k}\right)\right)$ is a spanning tree on $P$ in $M_{0}$. Therefore, we have

$$
\frac{L(T)}{L\left(\mathrm{MST}_{M_{0}}(P)\right)} \geq \eta\left(M_{0}\right)
$$

and thus $\rho\left(M_{0}\right) \geq \eta\left(M_{0}\right)$.

The following lemma will be used to prove Theorem 2 .

LEMMA 25. Any smooth flat polygonal surface can be immersed in the Euclidean plane $\boldsymbol{E}^{2}$.

PROOF. Let $D$ be a smooth flat polygonal surface with $n$ vertices. From the GaussBonnet theorem we have

$$
\sum_{i=1}^{n}\left(\pi-\theta_{i}\right)=2 \pi
$$

where $\theta_{i}$ is the inner angle at the vertex $p_{i} \in v(\partial D)$ for $i=1, \ldots, n$. Hence, we have 


$$
(n-2) \pi=\sum_{i=1}^{n} \theta_{i} .
$$

Since $n \geq 3$, there exists at least three vertices of $\partial D$ at which the inner angle are less than $\pi$. Let $p$ be such a vertex of $\partial D$ and $\theta$ the inner angle at $p$ which is less than $\pi$. Assume that $q_{1}$ and $q_{2}$ are the adjacent vertices of $p$ in $\partial D$. Consider the minimal curve $T$ connecting $q_{1}$ and $q_{2}$ in $D$. If it is a minimal geodesic segment $T\left(q_{1}, q_{2}\right)$ without broken point, then it is a diagonal of $D$. Otherwise, since there exists another vertex $q$ of $\partial D$ such that the inner angle at $q$ is less than $\pi$, we have $T \not \subset \partial D$. Therefore, there exists a diagonal of $D$. In any case we can find a diagonal of $D$. The polygonal surface is divided into two polygonal surfaces by the diagonal. The number of vertices of these polygonal surfaces are less than $n$. By mathematical induction we prove that $D$ has a triangulation by diagonals of $D$. Using this triangulation we construct an immersion from $D$ into the Euclidean plane $\boldsymbol{E}^{2}$, since each triangle domain in the triangulation is imbedded into $\boldsymbol{E}^{2}$.

7. Proof of Theorem 2. In this section we prove Theorem 2. Let $M$ be a complete Alexandrov surface with curvature bounded below by zero having no boundary and $M_{0}$ a strongly convex set. As stated after Proposition 24, we have $\rho\left(M_{0}\right) \geq \eta\left(M_{0}\right)$.

We prove $\eta\left(M_{0}\right) \geq \eta\left(\boldsymbol{E}^{2}\right)$. Let $D$ be an immersed polygonal surface in $M_{0}$. By Theorem 1 and Lemma 25 we construct a polygonal surface $\widetilde{D}$ from $D$ such that Int $\widetilde{D}$ is smooth and constant curvature zero and $\widetilde{D}$ is immersed in the Euclidean plane $\boldsymbol{E}^{2}$. Thus, we have

$$
\frac{L(\operatorname{SMT}(D))}{L(\operatorname{MST}(D))} \geq \frac{L(\operatorname{SMT}(\widetilde{D}))}{L(\operatorname{MST}(\widetilde{D}))} \geq \eta\left(\boldsymbol{E}^{2}\right) .
$$

This shows $\eta\left(M_{0}\right) \geq \eta\left(\boldsymbol{E}^{2}\right)$.

It remains to prove that the Steiner ratio of $M_{0}$ is less than or equal to $\sqrt{3} / 2$. In order to do this, we will find a sequence of geodesic triangles with vertex set $P(\varepsilon)=\{p(\varepsilon), q(\varepsilon), r(\varepsilon)\}$ of points in $M$ such that

$$
\frac{L\left(\operatorname{SMT}_{M}(P(\varepsilon))\right)}{L\left(\operatorname{MST}_{M}(P(\varepsilon))\right)} \rightarrow \frac{\sqrt{3}}{2} \quad \text { as } \quad \varepsilon \rightarrow 0 .
$$

Let $p, q$ and $r$ be points in $M_{0}$ such that its Steiner minimum tree has a Steiner point $w$. Take points $p(\varepsilon) \in T(w, p), q(\varepsilon) \in T(w, q)$ and $r(\varepsilon) \in T(w, r)$ such that $d(w, p(\varepsilon))=$ $d(w, q(\varepsilon))=d(w, r(\varepsilon))=\varepsilon$. Then, we have

$$
\frac{\varepsilon}{d(p(\varepsilon), q(\varepsilon))} \rightarrow \frac{1}{\sqrt{3}}
$$

as $\varepsilon \rightarrow+0$, since $d(p(\varepsilon), q(\varepsilon))^{2} \fallingdotseq 2 \varepsilon^{2}(1-\cos \omega(p(\varepsilon), q(\varepsilon)))$ and $\omega(p(\varepsilon), q(\varepsilon)) \rightarrow 2 \pi / 3$ as $\varepsilon \rightarrow+0$. Here $\fallingdotseq$ means that the left-hand side is approximated by the right-hand side (see [3, 2.9 Proposition in p.22]). In the same way we have

$$
\frac{\varepsilon}{d(q(\varepsilon), r(\varepsilon))} \rightarrow \frac{1}{\sqrt{3}} \quad \text { and } \quad \frac{\varepsilon}{d(r(\varepsilon), p(\varepsilon))} \rightarrow \frac{1}{\sqrt{3}}
$$


as $\varepsilon \rightarrow+0$. Since the Steiner minimum tree for $P(\varepsilon)=\{p(\varepsilon), q(\varepsilon), r(\varepsilon)\}$ is not longer than $T(w, p(\varepsilon)) \cup T(w, q(\varepsilon)) \cup T(w, r(\varepsilon))$ and a minimum spanning tree is the union of two shorter sides of the triangle $\triangle_{1}(p(\varepsilon) q(\varepsilon) r(\varepsilon))$, we see that

$$
\limsup _{\varepsilon \rightarrow+0} \frac{L(\operatorname{SMT}(P(\varepsilon)))}{L(\operatorname{MST}(P(\varepsilon)))} \leq \frac{\sqrt{3}}{2}
$$

This completes the proof of Theorem 2 .

\section{REFERENCES}

[1] A. D. Alexandrov, Selected Works Part II: Intrinsic Geometry of Convex Surfaces, Chapman and Hall/CRC, 2003.

[2] Yu. Burago, M. Gromov and G. Perelman, A. D. Alexandrov spaces with curvature bounded below, Russian Math. Surveys 47 (1992), 1-58.

[ 3 ] M. R. Bridson And A. Haefliger, Metric spaces of non-positive curvature, Springer, New York, 1999.

[4] H. Busemann, The geometry of geodesics, Academic Press, New York, 1955.

[5] J. Cheeger And D. G. Ebin, Comparison theorems in Riemannian geometry, AMS Chelsea Publishing, Providence, 2007.

[6] D. Z. Du And F. K. Hwang, The Steiner ratio conjecture of Gilbert and Pollak is true, Proc. Nat. Acad. Sci. U.S.A. 87 (1990), 9464-9466.

[ 7 ] E. N. Gilbert And H. O. Pollak, Steiner minimal trees, SIAM J. Appl. Math. 16 (1968), 1-29.

[ 8 ] N. InNAmi, Jacobi vector field along geodesics in glued Riemannian manifolds, Nihonkai Math. J. 12 (2001), 101-112.

[ 9 ] N. InNAMI, Compression theorems for surfaces and their applications, J. Math. Soc. Japan 59 (2007), 825835.

[10] N. Innami And B. H. Kim, Steiner ratio for hyperbolic surfaces, Proc. Japan Acad. Ser. A Math. Sci. 82 (2006), 77-79.

[11] N. Innami, B. H. Kim, Y. Mashiko and K. Shiohama, The Steiner ratio conjecture of Gilbert-Pollak may still be open, Algorithmica 57 (2010), 869-872.

[12] A. O. Ivanov And A. A. Tuzhilin, Immersed polygons and their diagonal triangulations, Izv. Math. 72 (2008), 63-90.

[13] A. O. Ivanov, A. A. Tuzhilin And D. Cieslik, Steiner ratio for manifolds, Math. Notes 74 (2003), $367-$ 374.

[14] J. H. Rubinstein And D. A. Thomas, A variational approach in the Steiner network problem, Ann. Oper. Res. 33 (1991), 481-499.

[15] J. H. Rubinstein And J. F. Weng, Compression theorems and Steiner ratio on spheres, J. Combin. Optimization 1 (1997), 67-78.

[16] M. TAKiguCHI, The index form of a geodesic on a glued Riemannian space, Nihonkai Math. J. 19 (2000), 167-202.

[17] M. TAKIGUChI, An extension of Rauch comparison theorem to glued Riemannian spaces, Tsukuba J. Math. 26 (2002), 313-338.

[18] S. TAmura AND N. InNAmI, Steiner ratios for length spaces having ends, Nihonkai Math. J. 19 (2008), 105-110.

[19] P. O. DE WET, Geometric Steiner minimal trees, Ph.D thesis, University of South Africa, 2008. 
Graduate School of Science And Technology NiIgATA UNIVERSITY

NIIGATA, 950-2181

JAPAN

E-mail address: f09n005e@mail.cc.niigata-u.ac.jp
Department OF MATHEMATICS

FACULTY OF SCIENCE

NIIGATA UNIVERSITY

NiIGATA, 950-2181

JAPAN

E-mail address: innami@math.sc.niigata-u.ac.jp 